\title{
Hot subdwarfs from the ESO Supernova la Progenitor Survey ${ }^{\star}$
}

\section{Atmospheric parameters and cool companions of sdB stars}

\author{
T. Lisker ${ }^{1,2}$, U. Heber ${ }^{1}$, R. Napiwotzki ${ }^{1,3}$, N. Christlieb ${ }^{4}$, Z. Han ${ }^{5}$, D. Homeier ${ }^{6}$, and D. Reimers ${ }^{4}$ \\ ${ }^{1}$ Dr.-Remeis-Sternwarte, Astronomisches Institut der Universität Erlangen-Nürnberg, Sternwartstr. 7, 96049 Bamberg, \\ Germany \\ e-mail: lisker@sternwarte.uni-erlangen.de \\ 2 Institute of Astronomy, ETH Zürich, Department of Physics, HPF D8, ETH Hönggerberg, 8093 Zürich, Switzerland \\ 3 Department of Physics \& Astronomy, University of Leicester, University Road, Leicester LE1 7RH, UK \\ ${ }^{4}$ Hamburger Sternwarte, Universität Hamburg, Gojenbergsweg 112, 21029 Hamburg, Germany \\ 5 The Yunnan Observatory, Academia Sinica, Kunming 650011, PR China \\ ${ }^{6}$ Department of Physics and Astronomy, University of Georgia, Athens, GA 30602-2451, USA
}

Received 9 February 2004 / Accepted 27 August 2004

\begin{abstract}
We present the analysis of a high-resolution, high-quality sample of optical spectra for 76 subdwarf B (sdB) stars from the ESO Supernova Ia Progenitor Survey (SPY, Napiwotzki et al. 2001). Effective temperature, surface gravity, and photospheric helium abundance are determined simultaneously by fitting the profiles of hydrogen and helium lines using synthetic spectra calculated from LTE and NLTE model atmospheres. We perform a detailed comparison of our measurements with theoretical calculations, both for single star evolution and for binary population synthesis models of close binary evolution. The luminosity evolution given by the standard EHB evolutionary tracks from Dorman et al. (1993) shows an overall agreement in shape with our observations, although a constant offset in luminosity exists. The various simulation sets for binary formation channels of sdB stars calculated by Han et al. (2003) are compared individually to our data for testing our current understanding of $\mathrm{sdB}$ formation processes and the physical effects involved. The best-matching sets manage to reproduce the observed sdB distribution in the temperature-gravity-plane well. However, they do not match the observed cumulative luminosity function, indicating that theoretical improvement is necessary. We also investigate composite-spectrum objects showing clear signatures of a cool companion with optical and infrared photometry. These stars have cool main sequence companions of spectral types F to K. Typical helium abundances of composite and non-composite sdB stars do not differ.
\end{abstract}

Key words. binaries: spectroscopic - stars: abundances - stars: atmospheres - stars: fundamental parameters stars: horizontal-branch - subdwarfs

\section{Introduction}

Early studies provided evidence that subdwarf B (sdB) stars are core helium burning stars with a canonical mass of $M \approx$ $0.5 M_{\odot}$, and a very thin hydrogen envelope $\left(M_{\text {env }}<0.01 M_{\odot}\right)$, placing them on the very hot end of the horizontal branch (HB), the so-called extreme horizontal branch (EHB). Unlike the typical post-HB evolution, these objects do not ascend the asymptotic giant branch (AGB) after core helium exhaustion, since hydrogen is not burned continuously in a shell due to the very low envelope mass. Instead, they evolve more or less directly to the white dwarf stage. Proposed formation scenarios are based on a late core helium flash in single star evolution (e.g. D'Cruz et al. 1996) or on mass transfer in close binary systems (e.g.

* Based on observations collected at the Paranal Observatory of the European Southern Observatory for program No. 165.H-0588(A) and 167.D-0407(A).
Mengel et al. 1976); furthermore, the merger of two helium white dwarfs (He-WDs) could be the origin of sdB stars (Iben 1990).

Today, more than $200 \mathrm{sdB}$ stars have been analyzed for atmospheric parameters (e.g. Saffer et al. 1994; Maxted et al. 2001; Edelmann et al. 2003), and this interpretation still seems to be valid in general: effective temperature and surface gravity of the observed sdBs mostly lie within the theoretically determined start and end points for core helium burning on the EHB, zero-age EHB (ZAEHB) and terminal-age EHB (TAEHB). However, the extensive theoretical study of binary formation mechanisms of sdB stars by Han et al. (2003, hereafter HPMM) shows that a huge number of sdB stars may be missing in observational surveys due to selection effects. These are primarily caused by main sequence companions that outshine the sdBs (main sequence spectral type $\mathrm{A}$ and earlier) or appear as composite spectrum objects (main sequence type $\mathrm{F}$ to $\mathrm{K}$ ). 
The latter have mostly been set aside because their spectral analysis is rendered difficult by the companion spectrum.

More than ten years after the first quantitative estimate of the contribution of different binary channels to the population of sdB stars (Tutukov \& Yungelson 1990), it is clear from the observations of Maxted et al. (2001) that close binary evolution is indeed of great importance to $\mathrm{sdB}$ formation processes, since they find that two thirds of their observed sdB stars are members of close binaries. The variety of physical parameters involved in those processes led HPMM to produce twelve simulation sets for sdB stars that formed in several evolutionary channels, i.e. by stable Roche lobe overflow (RLOF), common envelope (CE) ejection, or merging of two He-WDs. In comparing them with an observational sample providing good quality in atmospheric parameters, it should be possible to constrain the simulations' parameter range and to draw conclusions about the relative importance of each formation channel.

The ESO Supernova Ia Progenitor Survey (SPY, Napiwotzki et al. 2001) provides such a sample, since the ambitious project obtained high-resolution optical spectra of over 1000 white dwarf candidates, containing some 140 previously misclassified hot subdwarfs of various types. Earlier analyses of sdBs (e.g. Saffer et al. 1994; Maxted et al. 2001; Edelmann et al. 2003) were limited by the lower resolution of their spectra, sometimes drawn from inhomogeneous data sets. In many cases the wavelength coverage was incomplete as well, e.g. not all Balmer lines were included. Here we present the quantitative analysis of an unprecedented homogeneous set of high-resolution, high-quality spectra with large wavelength coverage, all of which makes it an excellent means for testing and increasing the knowledge of $\mathrm{sdB}$ formation and evolution.

Our report on the analysis of $76 \mathrm{sdB}$ stars from the SPY sample is outlined as follows. In Sect. 2, we describe the observations and data reduction of our objects. Section 3 contains information on the model atmospheres and the fit procedure used to determine atmospheric parameters, as well as on the examination of the $\mathrm{H} \alpha$ line and of spectral lines from cool companions. In Sect. 4, we analyze the sdB stars showing no spectral signatures of a cool companion and compare them to earlier studies. The composite-spectrum objects are investigated in Sect. 5, where we attempt to draw conclusions about the nature and spectral types of the companions. Finally, in Sect. 6, a detailed comparison of our observations with calculations for $\mathrm{sdB}$ formation and evolution is presented.

\section{Observations and data reduction}

Observations were obtained at the ESO Very Large Telescope with UT2 (Kueyen) equipped with the UV-Visual Echelle Spectrograph (UVES, Dekker et al. 2000). A slit width of 2'.1 was used, resulting in a spectral resolution of $18500(0.36 \AA$ at $\mathrm{H} \alpha$ ) or better. Wavelength coverage of $3300-6650 \AA$ is achieved, with gaps at 4500-4600 $\mathrm{A}$ and 5600-5700 $\mathrm{A}$. For most of the stars, two exposures in different nights were taken, since SPY was originally intended to search for RV-variable objects. The spectra were then reduced with a procedure developed by Karl (in prep.) using the ESO MIDAS software package, partly based on the UVES online reduction facility.
After accounting for cosmic ray hits and bad CCD pixels, bias and interorder background were subtracted, followed by order extraction for sky background, flatfield, and object. For each extracted one dimensional order, sky background subtraction and flatfielding were performed, and finally the orders were merged, resulting in three partial spectra separated by the gaps mentioned above. Those spectral parts are hereafter referred to as blue, lower red, and upper red part, respectively. Finally, each of them was divided by a smoothed spectrum of a DC white dwarf, which by definition shows no spectral features at all and therefore provides an excellent means of correcting for the instrumental response.

The spectra were then convolved with a Gaussian of $1.0 \AA$ $F W H M$ and rebinned to $0.4 \AA$ stepsize. A signal-to-noise ratio $(S / N)$ was defined and calculated for the blue parts of the spectra, since they contain most of the lines used for fitting, as the ratio of mean flux to standard deviation in a continuum area. The median value is 178 , all except one have values of $S / N \geq 10$, nine spectra even have $S / N>1000$.

Photometric data are available for all of our objects. $B$ magnitudes of most of the stars are photographic measurements provided by the Hamburg/ESO Survey (HES, Wisotzki et al. 1996), the Hamburg Quasar Survey (HQS, Hagen et al. 1995), and McCook \& Sion (1999). For nine HES stars, $B$ and $V$ values were taken from the CCD photometry of Altmann et al. (2004). In some cases, only $V$ or Strömgren- $y$ magnitudes were available. $J$ magnitudes have been drawn from the Two Micron All Sky Survey (2MASS) archive.

\section{Spectral analysis and classification}

\subsection{Model atmospheres and line profile fitting}

Effective temperatures $\left(T_{\text {eff }}\right)$, surface gravities $(\log (g))$, and helium abundances $\left(y=N_{\mathrm{He}} / N_{\mathrm{H}}\right)$ were determined by fitting simultaneously each hydrogen and helium line to synthetic model spectra, using a procedure developed by R. Napiwotzki (Napiwotzki et al. 1999) based on Saffer et al. (1994). Three different sets of models were used, where the last two make use of PRO2 (Werner \& Dreizler 1999):

1. A grid of metal line-blanketed LTE model atmospheres with solar abundance (Heber et al. 2000) for stars with $T_{\text {eff }}<32000 \mathrm{~K}$.

2. A grid of partially line-blanketed NLTE model atmospheres (Napiwotzki 1999) for stars with $T_{\text {eff }}>32000 \mathrm{~K}$.

3. A grid of partially line-blanketed NLTE model atmospheres for helium rich objects, used for two stars with $\log (y)>-1$ (updated version of Dreizler et al. 1990).

A typical LTE and NLTE fit is shown in Fig. 1. For each line, the continuum level is determined and normalized to 1 , in order to compare it to the synthetic spectrum. $\mathrm{H} \alpha$ was never included in the parameter determination itself, but was kept in the final plot for examining possible deviations from the model $\mathrm{H} \alpha$ line. The core of $\mathrm{H} \alpha$ is sensitive to NLTE effects because it is formed in the very outer parts of the atmosphere. Therefore, LTE models often fail to reproduce the observations. This is evident in 
the example given for a LTE analysis in Fig. 1a. NLTE analyses, however, usually are able to reproduce the $\mathrm{H} \alpha$ profiles of sdB stars very well (Heber et al. 2003), as exemplified in Fig. 1b. We will discuss the $\mathrm{H} \alpha$ lines of the programme stars in more detail in Sect. 3.4.

\subsection{Spectral classification}

The hot subdwarfs had been selected from the SPY data by using Balmer line widths as an indicator for surface gravity and by performing a line profile fit where visual inspection did not yield unambigous results. After having excluded objects that clearly have helium-dominated atmospheres, the presence and strength of the He II lines was used for distinguishing between $\mathrm{sdB}, \mathrm{sdOB}$, and sdO stars. In this paper we present analyses of the sdB and sdOB stars from SPY, subsequently referred to as sdB stars. This sample comprises $76 \mathrm{sdBs}$ with effective temperatures between $20000 \mathrm{~K}<T_{\text {eff }}<38000 \mathrm{~K}$, gravities in the range $4.8<\log (g)<6.0$, and helium abundances between $-4.0 \leq \log (y)<-0.8$ (see Table 1). 51 program stars are objects from HES, 9 from HQS, 14 from McCook \& Sion (1999), and 2 are from the list of white dwarf central stars of planetary nebulae by Napiwotzki (1999), see Table 1.

\subsection{Spectroscopic and photometric signatures of cool companions}

Our sample of sdB stars includes many objects where signatures of a cool companion can be seen in the spectrum. In some of them, the companion shows a large contribution to the total flux even in the blue part of the spectrum, causing a broad Ca K line (3933 $\AA$ ) as opposed to the frequently occurring narrow interstellar $\mathrm{Ca} \mathrm{K}$ line. In addition, $G$-band absorption was taken as a clear companion signature. For most objects though, the contribution of the companion is best visible in the lower red part of the spectrum, where the $\mathrm{Mg}$ I triplet at $5167 \AA, 5173 \AA$, and $5184 \AA$ is the most striking feature. Since the latter is not known to occur in $\mathrm{sdB}$ atmospheres nor in the interstellar medium, we used it as a tracer to find even very faint companions in sdB spectra that appeared to be non-composite at first glance. These examinations yielded altogether $19 \mathrm{sdB}$ stars with a cool companion contributing to the spectrum (see Table 1). They are treated separately (cf. Sect. 5) from the noncomposite objects, because the results from line profile fitting cannot be considered reliable.

Since our spectra have not been flux calibrated, it was not possible to verify these findings by making use of the flux distribution in the upper red part, which should otherwise clearly show the contribution of the companion. Instead, we retrieved infrared photometric data for our stars from the 2MASS database to examine the quality of the $\mathrm{Mg}$ I triplet as tracer for cool companions. 67 of our objects show a detection at least in $J$ and are unambiguously identified, whereas 9 show no detection at all and cannot be mistaken with nearby sources. In the latter cases, we derived $J<16.5$ as a conservative estimate of the detection limit from the fluxes of nearby sources in the field of view.
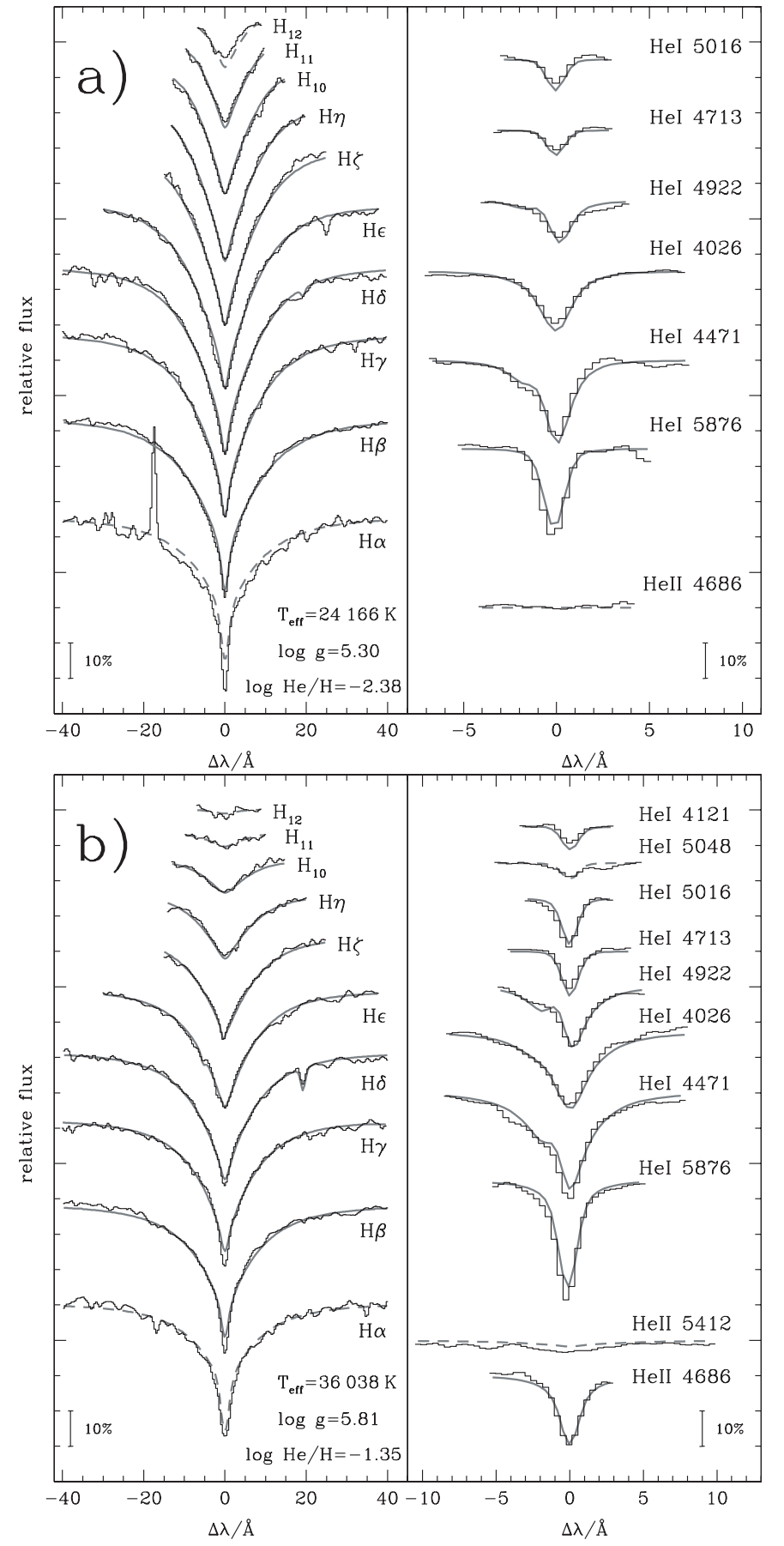

Fig. 1. a) Sample fit of a relatively cool sdB (GD 687) using synthetic spectra calculated from LTE model atmospheres. b) Sample fit of a relatively hot $\mathrm{sdB}$ (GD 619) using synthetic spectra calculated from NLTE model atmospheres. In both panels, the observed spectra are shown as histograms. When a spectral line was used for parameter determination, model spectra are plotted as solid grey lines, otherwise as dashed grey lines. The continua of observed and model spectrum were scaled to the same level on both sides of the line. The resulting atmospheric parameter values are displayed in the figure.

The 2MASS observations are complete ${ }^{1}$ down to $J \leq 15$. 8 , $H \leq 15$ m 1 and $K \leq 14$. 3 . Since more than half of our sample is

\footnotetext{
${ }^{1}$ http://www.ipac.caltech.edu/2mass/overview/ about2mass.html
} 
Table 1. Results of our spectral analysis. $T_{\text {eff }}, \log (g)$ and $\log (y)$ are mean values from two or more exposures, if possible. Luminosity in units of solar luminosity is calculated by assuming $M_{\mathrm{sdB}}=0.5 M_{\odot}$.

\begin{tabular}{|c|c|c|c|c|c|c|c|c|c|c|c|}
\hline Object & $\begin{array}{l}T_{\text {eff }} \\
\mathrm{K}\end{array}$ & $\begin{array}{l}\log (g) \\
\mathrm{cm} \mathrm{s}^{-2}\end{array}$ & $\log (y)$ & $\begin{array}{l}\log (L) \\
L_{\text {edd }}\end{array}$ & $\begin{array}{l}\log (L) \\
L_{\odot}\end{array}$ & $\begin{array}{l}M_{V} \\
\text { mag }\end{array}$ & $\begin{array}{l}d \\
\mathrm{kpc}\end{array}$ & $\begin{array}{l}\text { RA (J2000) } \\
\text { hh:mm:ss.ss }\end{array}$ & $\begin{array}{l}\operatorname{Dec}(\mathbf{J} 2000) \\
\pm \mathrm{dd}: m m: s s . s\end{array}$ & $\begin{array}{l}B \\
\text { mag }\end{array}$ & Note \\
\hline EGB 5 & 34060 & 5.85 & -2.77 & -2.84 & 1.37 & 4.50 & 0.7 & $08: 11: 12.77$ & $+10: 57: 16.8$ & $13.8^{c}$ & 7 \\
\hline HE 0007-2212 & 28964 & 5.68 & $\leq-4.00$ & -2.95 & 1.25 & 4.42 & 1.4 & 00:09:45.91 & $-21: 56: 14.4$ & 15.0 & 10 \\
\hline HE $0016+0044$ & 28264 & 5.38 & -2.66 & -2.70 & 1.51 & 3.72 & 1.7 & $00: 18: 43.59$ & $+01: 01: 22.5$ & 14.8 & \\
\hline HE 0019-5545 & 35662 & 5.86 & -1.44 & -2.76 & 1.44 & 4.45 & 2.2 & $00: 21: 27.68$ & $-55: 29: 12.3$ & 15.8 & \\
\hline HE 0101-2707 & 35568 & 5.97 & -0.92 & -2.88 & 1.32 & 4.73 & 1.2 & 01:03:43.73 & $-26: 51: 53.8$ & 15.1 & 10 \\
\hline HE $0123-3330$ & 36602 & 5.87 & -1.49 & -2.73 & 1.47 & 4.45 & 1.6 & $01: 25: 22.46$ & $-33: 15: 11.4$ & 15.2 & 10 \\
\hline HE $0135-6150$ & 27020 & 5.59 & -2.47 & -2.99 & 1.22 & 4.34 & 2.8 & 01:37:30.08 & $-61: 34: 57.8$ & 16.3 & \\
\hline HE 0136-2758 & 28202 & 5.47 & $\leq-4.00$ & -2.79 & 1.41 & 3.95 & 2.8 & 01:39:14.46 & $-27: 43: 21.7$ & 15.94 & 5,10 \\
\hline HE 0151-3919 & 20841 & 4.83 & -2.07 & -2.68 & 1.53 & 2.94 & 1.9 & 01:53:11.18 & $-39: 04: 18.2$ & 14.09 & 5,9 \\
\hline HE $0207+0030$ & 31414 & 5.83 & -1.83 & -2.95 & 1.25 & 4.63 & 1.4 & 02:10:14.97 & $+00: 45: 02.1$ & 15.1 & 8 \\
\hline HE $0230-4323$ & 31552 & 5.60 & -2.58 & -2.72 & 1.48 & 4.04 & 0.9 & 02:32:54.66 & $-43: 10: 27.9$ & 13.56 & 5 \\
\hline HE 0306-0309 & 26710 & 5.79 & $\leq-4.00$ & -3.20 & 1.01 & 4.86 & 1.9 & 03:08:40.80 & $-02: 58: 02.8$ & 16.1 & \\
\hline HE 0321-0918 & 25114 & 5.67 & -3.02 & -3.18 & 1.02 & 4.69 & 1.3 & 03:23:45.85 & $-09: 08: 15.6$ & 15.2 & \\
\hline HE 0415-2417 & 32768 & 5.12 & -2.44 & -2.18 & 2.03 & 2.76 & 5.6 & $04: 17: 31.49$ & $-24: 09: 50.7$ & 16.2 & \\
\hline HE 0513-2354 & 26758 & 5.50 & -2.33 & -2.91 & 1.29 & 4.14 & 2.4 & 05:15:15.30 & $-23: 51: 09.5$ & 15.8 & \\
\hline HE $0532-4503$ & 25710 & 5.33 & -3.07 & -2.81 & 1.40 & 3.79 & 2.8 & $05: 33: 40.51$ & $-45: 01: 35.3$ & 15.84 & 5 \\
\hline HE 0539-4246 & 23279 & 5.51 & -3.91 & -3.16 & 1.05 & 4.43 & 1.2 & 05:41:06.71 & $-42: 45: 31.9$ & 14.60 & 5 \\
\hline HE 0929-0424 & 29602 & 5.69 & -2.01 & -2.92 & 1.28 & 4.40 & 1.9 & 09:32:02.15 & $-04: 37: 37.8$ & 15.4 & \\
\hline HE 1021-0255 & 35494 & 5.81 & -1.46 & -2.73 & 1.48 & 4.33 & 1.7 & $10: 24: 18.06$ & $-03: 10: 38.8$ & 15.3 & 1,8 \\
\hline HE 1033-2353 & 36204 & 5.76 & -1.46 & -2.64 & 1.56 & 4.19 & 2.6 & $10: 36: 07.23$ & $-24: 08: 35.4$ & 16.0 & 9 \\
\hline HE $1038-2326$ & $30573^{a}$ & $5.21^{b}$ & $-1.73^{b}$ & & & & & $10: 40: 36.97$ & $-23: 42: 39.4$ & 15.9 & $1,2,9$ \\
\hline HE 1047-0436 & 30280 & 5.71 & -2.35 & -2.91 & 1.30 & 4.41 & 1.3 & $10: 50: 26.93$ & $-04: 52: 35.8$ & 14.7 & 8 \\
\hline HE $1050-0630$ & 34501 & 5.79 & -1.40 & -2.76 & 1.45 & 4.33 & 0.9 & $10: 53: 26.52$ & $-06: 46: 15.6$ & 14.0 & 8 \\
\hline HE $1140-0500$ & $34522^{a}$ & $4.97^{b}$ & $-2.62^{b}$ & & & & & $11: 42: 57.85$ & $-05: 17: 14.1$ & 14.8 & 2,8 \\
\hline HE 1200-0931 & 33419 & 5.78 & -1.88 & & & & & $12: 03: 21.77$ & $-09: 48: 06.6$ & 16.2 & 3 \\
\hline HE $1221-2618$ & $32606^{a}$ & $5.51^{b}$ & $-1.84^{b}$ & & & & & $12: 24: 32.72$ & $-26: 35: 17.1$ & 14.7 & 2,9 \\
\hline HE $1254-1540$ & 29700 & 5.63 & $\leq-4.00$ & & & & & $12: 57: 19.36$ & $-15: 56: 22.8$ & 15.2 & 3,9 \\
\hline HE $1309-1102$ & $27109^{a}$ & $5.36^{b}$ & $-2.46^{b}$ & & & & & $13: 12: 02.39$ & $-11: 18: 16.3$ & 16.1 & 2 \\
\hline HE $1352-1827$ & $35674^{a}$ & $5.53^{b}$ & $-1.77^{b}$ & & & & & $13: 55: 26.67$ & $-18: 42: 09.4$ & 16.0 & 2,9 \\
\hline HE $1407+0033$ & 37309 & 5.54 & -2.99 & -2.38 & 1.83 & 3.61 & 2.4 & $14: 10: 20.73$ & $+00: 18: 54.6$ & 15.5 & 4,8 \\
\hline HE 1415-0309 & 29520 & 5.56 & -2.87 & -2.80 & 1.41 & 4.09 & 3.0 & $14: 18: 20.93$ & $-03: 22: 54.1$ & 16.3 & \\
\hline HE $1419-1205$ & 34171 & 5.71 & -1.65 & & & & & $14: 22: 02.17$ & $-12: 19: 30.9$ & 16.2 & 3 \\
\hline HE $1421-1206$ & 29570 & 5.53 & $\leq-4.00$ & -2.76 & 1.45 & 4.01 & 1.9 & $14: 24: 08.81$ & $-12: 20: 21.5$ & 15.1 & 8 \\
\hline HE $1422-1851$ & $33896^{a}$ & $5.19^{b}$ & $-3.07^{b}$ & & & & & $14: 24: 48.65$ & $-19: 05: 01.3$ & 16.3 & $1,2,9$ \\
\hline HE $1441-0558$ & $36396^{a}$ & $5.79^{b}$ & $-1.63^{b}$ & & & & & $14: 44: 12.11$ & $-06: 10: 44.7$ & 14.4 & 2 \\
\hline HE $1448-0510$ & 34760 & 5.53 & -3.41 & -2.48 & 1.72 & 3.66 & 1.5 & $14: 51: 13.13$ & $-05: 23: 16.9$ & 14.4 & 4,8 \\
\hline HE 1450-0957 & 34563 & 5.79 & -1.29 & -2.75 & 1.45 & 4.32 & 1.7 & $14: 53: 24.19$ & $-10: 09: 21.9$ & 15.1 & 9 \\
\hline HE 1459-0234 & & & & & & & & $15: 02: 12.29$ & $-02: 46: 00.9$ & 14.9 & 2 \\
\hline HE 1519-0708 & 34498 & 5.73 & -1.52 & -2.70 & 1. & 4.18 & 2.3 & $15: 21: 53.20$ & $-07: 19: 23.6$ & 15.6 & 8 \\
\hline HE 2135-3749 & 29924 & 5.87 & -2.45 & -3.08 & 1.13 & 4.83 & 0.7 & 21:38:44.18 & $-37: 36: 15.1$ & 13.70 & 5 \\
\hline HE $2150-0238$ & 29846 & 5.90 & -2.36 & -3.12 & 1.09 & 4.91 & 1.7 & 21:52:35.81 & $-02: 24: 31.6$ & 15.8 & \\
\hline HE 2151-1001 & 34984 & 5.70 & -1.60 & -2.64 & 1.57 & 4.07 & 2.2 & 21:54:31.49 & $-09: 47: 30.5$ & 15.6 & 4 \\
\hline HE $2156-3927$ & $27995^{a}$ & $5.50^{b}$ & $-2.35^{b}$ & & & & & 21:59:35.53 & $-39: 13: 15.3$ & 14.26 & $2,5,11$ \\
\hline HE 2201-0001 & 27062 & 5.51 & -3.29 & -2.90 & 1.31 & 4.14 & 2.7 & $22: 04: 18.27$ & $+00: 12: 36.7$ & 16.0 & \\
\hline HE $2208+0126$ & 24277 & 5.67 & -2.98 & -3.25 & 0.96 & 4.75 & 1.4 & $22: 10: 45.47$ & $+01: 41: 35.4$ & 15.2 & 1,8 \\
\hline HE $2222-3738$ & 30248 & 5.69 & -3.65 & -2.88 & 1.32 & 4.36 & 1.3 & $22: 24: 56.50$ & $-37: 23: 30.7$ & 14.66 & 5 \\
\hline HE $2237+0150$ & 25606 & 5.38 & -1.92 & -2.86 & 1.34 & 3.92 & 2.7 & $22: 40: 14.38$ & $+02: 06: 31.3$ & 15.8 & 8 \\
\hline HE 2238-1455 & 30393 & 5.47 & -2.37 & -2.66 & 1.54 & 3.80 & 3.0 & $22: 41: 38.27$ & $-14: 39: 39.5$ & 16.0 & \\
\hline HE 2307-0340 & 23260 & 5.51 & -3.65 & -3.16 & 1.04 & 4.44 & 2.1 & 23:10:24.09 & $-03: 24: 02.3$ & 15.8 & \\
\hline HE $2322-0617$ & $28106^{a}$ & $5.50^{b}$ & $-1.93^{b}$ & & & & & $23: 25: 31.91$ & $-06: 01: 12.1$ & 15.7 & 2 \\
\hline HE 2322-4559 & $25512^{a}$ & $5.30^{b}$ & $-2.47^{b}$ & & & & & 23:25:09.05 & $-45: 43: 06.5$ & 15.5 & 2 \\
\hline HE 2349-3135 & 28520 & 5.44 & -3.84 & -2.74 & 1.47 & 3.86 & 2.6 & $23: 51: 43.63$ & $-31: 18: 52.9$ & 15.63 & 5,10 \\
\hline HS $1530+0542$ & & & & & & & & $15: 33: 10.74$ & $+05: 32: 26.8$ & 14.3 & 2,8 \\
\hline HS $1536+0944$ & $35114^{a}$ & $5.83^{b}$ & $-0.82^{b}$ & & & & & $15: 38: 42.88$ & $+09: 34: 42.8$ & 15.6 & $1,2,8$ \\
\hline
\end{tabular}


Table 1. Continued.

\begin{tabular}{|c|c|c|c|c|c|c|c|c|c|c|c|}
\hline Object & $\begin{array}{l}T_{\text {eff }} \\
\mathrm{K}\end{array}$ & $\begin{array}{l}\log (g) \\
\mathrm{cm} \mathrm{s}^{-2}\end{array}$ & $\log (y)$ & $\begin{array}{l}\log (L) \\
L_{\text {edd }}\end{array}$ & $\begin{array}{l}\log (L) \\
L_{\odot}\end{array}$ & $\begin{array}{l}M_{V} \\
\text { mag }\end{array}$ & $\begin{array}{l}d \\
\mathrm{kpc}\end{array}$ & $\begin{array}{l}\text { RA (J2000) } \\
\text { hh:mm:ss.ss }\end{array}$ & $\begin{array}{l}\text { Dec (J2000) } \\
\pm \text { dd:mm:ss.s }\end{array}$ & $\begin{array}{l}B \\
\mathrm{mag}\end{array}$ & Note \\
\hline HS $1710+1614$ & 34826 & 5.72 & -1.64 & -2.68 & 1.53 & 4.13 & 2.4 & $17: 13: 03.18$ & $+16: 10: 42.8$ & 15.7 & 4 \\
\hline HS $2033+0821$ & 32706 & 5.87 & -1.56 & -2.93 & 1.28 & 4.64 & 1.0 & $20: 35: 29.34$ & $+08: 31: 51.7$ & 14.4 & \\
\hline HS $2043+0615$ & 26157 & 5.28 & -2.38 & -2.73 & 1.48 & 3.63 & 3.4 & $20: 46: 20.86$ & $+06: 26: 24.4$ & 16.0 & \\
\hline HS $2125+1105$ & 32542 & 5.76 & -1.86 & & & & & $21: 27: 32.17$ & $+11: 18: 17.1$ & 16.4 & 3 \\
\hline HS $2216+1833$ & $34361^{a}$ & $5.51^{b}$ & $-1.70^{b}$ & & & & & $22: 18: 30.60$ & $+18: 48: 09.4$ & 13.8 & 2 \\
\hline HS $2357+2201$ & 27629 & 5.55 & -2.54 & -2.90 & 1.30 & 4.20 & 0.8 & 00:00:18.41 & $+22: 18: 03.0$ & 13.3 & 1 \\
\hline HS 2359+1942 & 31434 & 5.56 & -3.58 & -2.69 & 1.52 & 3.95 & 1.4 & 00:02:08.46 & $+19: 59: 12.8$ & 14.4 & 1,8 \\
\hline PHL 932 & 33644 & 5.74 & -1.64 & -2.75 & 1.46 & 4.25 & 0.4 & $00: 59: 56.65$ & $+15: 44: 13.6$ & $12.1^{c}$ & 6,8 \\
\hline \multicolumn{12}{|c|}{ sdB stars misclassified in the McCook $\mathcal{F}$ Sion (1999) catalog of white dwarfs: } \\
\hline CBS 275 & 29262 & 5.72 & -2.46 & -2.97 & 1.23 & 4.50 & 1.3 & 97.33 & $-27:$ & 14.8 & 9 \\
\hline GD 617 & & & & & & & & $00: 31: 13.06$ & $-27: 12: 54.4$ & $15.2^{d}$ & 2,10 \\
\hline GD 619 & 36097 & 5.82 & -1.33 & -2.71 & 1.49 & 4.34 & 0.9 & $00: 33: 53.88$ & $-27: 08: 23.6$ & 13.9 & 10 \\
\hline GD 687 & 24350 & 5.32 & -2.38 & -2.90 & 1.31 & 3.87 & 1.1 & $01: 10: 18.46$ & $-34: 00: 26.4$ & $14.1^{d}$ & 10 \\
\hline GD 1237 & & & & & & & & $23: 45: 26.69$ & $-15: 28: 38.6$ & 15.5 & 2 \\
\hline KUV 01542-0710 & 27760 & 5.44 & -2.91 & & & & & $01: 56: 42.42$ & $-06: 55: 40.2$ & $16.3^{c}$ & 3 \\
\hline PG $0258+184$ & $28092^{a}$ & $5.52^{b}$ & $-3.03^{b}$ & & & & & 03:01:12.87 & $+18: 40: 54.0$ & 15.3 & 2 \\
\hline PG 1207-032 & 35693 & 5.82 & -1.48 & -2.73 & 1.48 & 4.35 & 0.7 & 12:09:36.04 & $-03: 33: 08.0$ & $13.5^{d}$ & 1 \\
\hline PG 1549-001 & 28252 & 5.49 & -2.66 & -2.80 & 1.40 & 4.00 & 1.7 & $15: 52: 02.77$ & $-00: 04: 39.4$ & $15.2^{c}$ & \\
\hline PG $2122+157$ & $26015^{a}$ & $5.22^{b}$ & $-2.69^{b}$ & & & & & $21: 24: 54.89$ & $+15: 59: 03.6$ & 15.0 & 2 \\
\hline PHL 555 & 34126 & 5.77 & -1.36 & -2.76 & 1.45 & 4.30 & 0.8 & $23: 31: 49.97$ & $-28: 52: 53.1$ & $13.8^{c}$ & \\
\hline PHL 861 & 29668 & 5.50 & $\leq-4.00$ & -2.73 & 1.47 & 3.93 & 1.5 & 00:51:03.97 & $-20: 00: 00.3$ & $14.9^{d}$ & 10 \\
\hline SB 485 & 27738 & 5.51 & -2.50 & -2.85 & 1.36 & 4.09 & 0.6 & $01: 12: 11.65$ & $-26: 13: 27.9$ & 12.9 & 10 \\
\hline TON S 155 & $32318^{a}$ & $5.16^{b}$ & $-3.04^{b}$ & & & & & $00: 23: 59.35$ & $-23: 09: 53.5$ & $16.1^{d}$ & 2,10 \\
\hline
\end{tabular}

$a$ The given value is an upper limit due to the presence of a cool companion.

$b$ The given value is a lower limit due to the presence of a cool companion.

c Johnson $V$ magnitude

${ }^{d}$ Strömgren $y$ magnitude

$1=$ stars for which there was only one useful exposure.

2 = a cool companion shows $\mathrm{Mg}$ I in the spectrum, and possibly additional features.

3 = the presence of a companion was deduced solely from a flux contribution at $\mathrm{H} \alpha$. Atmospheric parameters have been determined from Balmer and helium lines in the blue part of the spectrum. Unlike for the stars with earlier type companions, the contribution of the companion in this spectral region is irrelevant, and the parameters can be regarded as reliable (for details see Sect. 5.1).

4 = single-lined objects showing peculiar $\mathrm{H} \alpha$ profiles.

5 = independently classified as sdB by Altmann et al. (2004).

6 = central star of the planetary nebula PN G 125.9-47.0.

7 = central star of the planetary nebula PN G 211.9+22.6.

8 = also in the Palomar-Green survey (PG, Green et al. 1986).

9 = also in the Edinburgh-Cape survey (EC, Stobie et al. 1987).

$10=$ also in the Montreal-Cambridge-Tololo survey (MCT, Demers et al. 1987).

11 = In Table 1 of Altmann et al. (2004), $V$ magnitudes of this star and HE 2156-1732 have to be interchanged (M. Altmann, priv. comm.).

fainter than $J=15 \cdot 5$, their $H$ and $K$ fluxes are of little use and were not considered. We based our analysis on $B-J$ colours, because $V$-band measurements are unavailable for most stars. In Fig. 2 we plot $B$ vs. $B-J$ for all 76 sdB stars. The apparently single $\mathrm{sdB}$ stars separate from objects with a cool companion at about $B-J \approx 0$, which nicely agrees with the detection of $\mathrm{Mg} \mathrm{I}$ in their spectra (grey circles in Fig. 2).

For companion spectral types later than K0, the difference in colours between an $\mathrm{sdB}+$ main sequence system and an sdB alone decreases and finally almost vanishes, because of the decreasing contribution of the companion to the total flux (cf. Sect. 5.1). Hence, there is a limit in spectral type above which an $\mathrm{sdB}+$ main sequence system may lie at $B-J<0$, and therefore cannot be identified as composite. Three stars with $B-J<0$ show Mg I as well, confirming the quality of the latter as an even better tracer for cool companions than colour values are.

\subsection{Peculiar $\mathrm{H} \alpha$ line profiles}

A close examination of the $\mathrm{H} \alpha$ line of the apparently noncomposite objects yielded ten stars with peculiar $\mathrm{H} \alpha$ profiles. They can be divided into two groups: five of them show a line core which is shallower than that of the corresponding model 


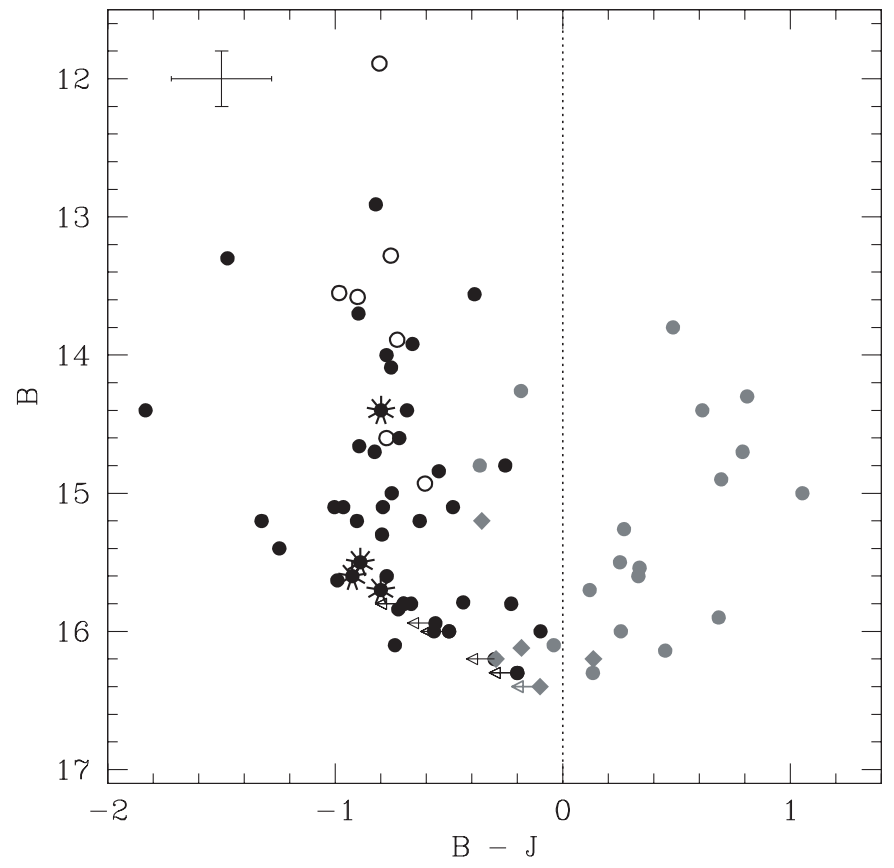

Fig. 2. Colour-magnitude diagram of $B$ versus $B-J$ for all 76 objects. Composite spectrum objects are plotted as grey circles if they are classified from the presence of the $\mathrm{Mg}$ I triplet in their spectra, or as grey diamonds if they are classified from the contribution of the companion to the $\mathrm{H} \alpha$ line profile (see text). Asterisks represent the four stars with peculiar $\mathrm{H} \alpha$ profiles. The dotted vertical line marks the value $B-J=0$ m 0 . For the stars marked as open symbols, we adopted $B \approx V-0.25$ or $B \approx y-0^{\mathrm{m}} \cdot 25$, because no $B$ measurements are available.

line, but has a normal line core shape. This peculiarity is seen in seven composite objects as well, suggesting that this is another hint of a cool companion. It is demonstrated in Fig. 3 where we compare synthetic $\mathrm{H} \alpha$ line profiles calculated from NLTE model atmospheres to observed ones for a typical sdB (Fig. 3a) and for a representative composite one showing this peculiarity (Fig. 3b). Atmospheric parameters have been derived for each star from other Balmer and helium lines in the blue part of its spectrum. It must be stressed that we did not fit the $\mathrm{H} \alpha$ line profile by a synthetic spectrum. As can be clearly seen, the $\mathrm{H} \alpha$ line profile in a typical sdB is quite well matched, whereas in the peculiar star it is too shallow. Since the cool companion adds some continuum flux, it dilutes the $\mathrm{H} \alpha$ profile of the sdB. Hence, after normalization, the $\mathrm{H} \alpha$ line depth is lower than it would be without the companion contribution, and therefore lower than that of the synthetic profile. This is exactly what we see in those stars. We subtracted an appropriate constant from the spectrum around $\mathrm{H} \alpha$. Now the corrected $\mathrm{H} \alpha$ profile is perfectly matched by the synthetic profile (see Fig. 3c). Since this is shown for a star that we already classified as composite, by subtracting a constant we automatically demonstrated that the contribution of the companion $\mathrm{H} \alpha$ line is negligible. Applying this treatment to the five non-composite stars leads to the same result, which we take as evidence for the presence of a cool companion (see Table 1), although other spectral features (i.e. Mg I) are not detected in these stars.
Since the companion contribution decreases with decreasing wavelength, it is understandable why in the blue and lower red part of the spectrum no cool star features can be seen. The companions are therefore expected to be of very late type, consistent with the colour-magnitude-diagram $(B, B-J)$ (Fig. 2): one star lies among the composite objects, the others lie among the single sdBs, but are very close to $B-J=0$, which we regard as the photometric detection limit for composite objects. As discussed in the previous subsection, this is exactly what one expects for very late type companions, thus supporting our conclusion. We summarize that 24 out of $76 \mathrm{sdB}$ stars show signatures of a cool companion, hereafter termed double-lined objects, even if the stars just discussed do not show any obvious companion lines. As mentioned above, they are treated separately from the 52 non-composite (single-lined) sdBs.

A second kind of peculiarity of the $\mathrm{H} \alpha$ profile is found in five other objects of our sample: the core is flat, and the inner wing is broader than predicted by the model, as seen in Fig. 4. One of these stars, EGB 5, is the central star of a planetary nebula. The $\mathrm{H} \alpha$ emission of its nebula may not have been correctly subtracted in the semi-automatic data reduction process. Close inspection of sdB spectra that we regard as normal (Fig. 3a) reveals that slight mismatches of the $\mathrm{H} \alpha$ line core occur even in those stars. However, these effects are so small that they could be caused by errors in the data reduction and/or the atmospheric models and are therefore considered as insignificant. Nevertheless, we are left with four stars with peculiar $\mathrm{H} \alpha$ profiles.

The colour values of this group of peculiar stars (Fig. 2, asterisks) clearly show that they cannot be composite objects. There is no significant line profile variation observed from one to the next exposure of the star. Time intervals range from $3 \mathrm{~d}$ to $1 \mathrm{yr}$. Although the observed line broadening could possibly be caused by rotation, convolution of the synthetic spectra with rotational profiles does not lead to acceptable fits for any hydrogen or helium line. Even for $\mathrm{H} \alpha$ itself, the match is still not satisfying, implying that other physical effects are present.

Since at this point there is no interpretation for this $\mathrm{H} \alpha$ profile peculiarity, we proceed with our analysis, and will distinguish those stars from the rest of single-lined objects wherever applicable.

\section{Single-lined objects}

\subsection{Error determination}

The statistical 1- $\sigma$-errors from the fit procedure are typically lower than $100 \mathrm{~K}, 0.02 \mathrm{dex}$, and $0.04 \mathrm{dex}$ for $T_{\text {eff }}, \log (g)$, and $\log (y)$, respectively. However, despite the high resolution and the low noise level of our data, we do not believe these to be the "true errors". To obtain a good estimate for the latter, we take advantage of the SPY observing strategy: as mentioned above, at least two exposures were taken for most of the stars. Hence, for determining reliable errors we used the distribution of differences in the fit results of individual exposures of each object (Fig. 5). Fitting this distribution by a Gaussian, we adopt the width of the latter as the error of the fit results of individual exposures. This is a quite conservative treatment intended 

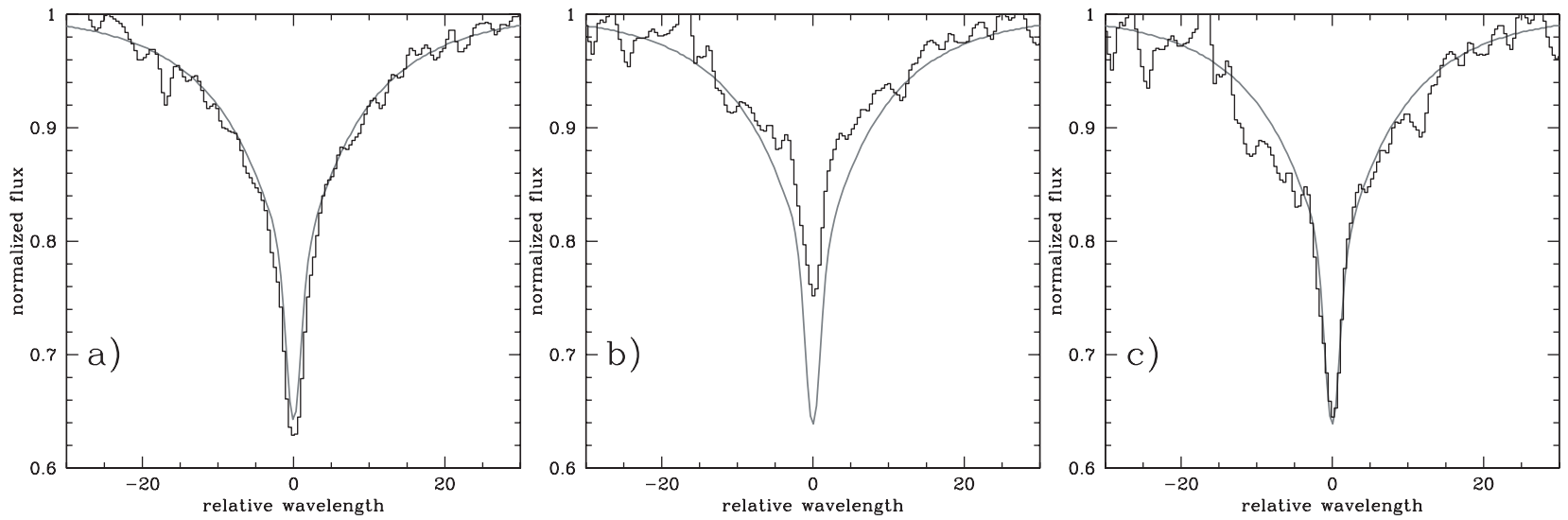

Fig. 3. Comparison of synthetic $\mathrm{H} \alpha$ line profiles calculated from NLTE model atmospheres to observed ones (see text). a) GD 619: a typical sdB, b) HE 1441-0558: a composite sdB star, c) HE 1441-0558: after correction for continuum light from the cool companion.

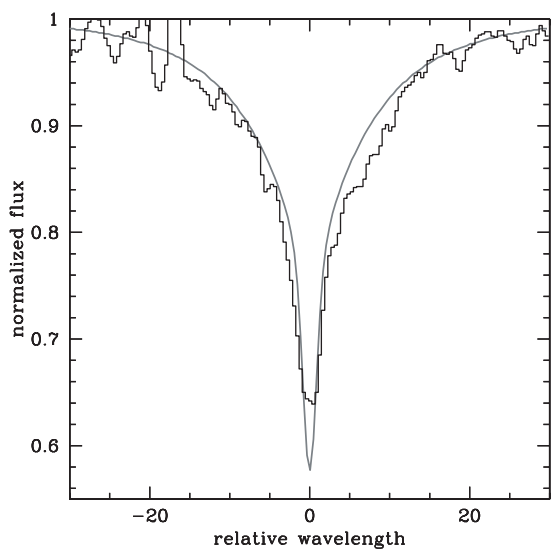

Fig. 4. Same as Fig. 3, but here we show the second kind of $\mathrm{H} \alpha$ profile peculiarity we observed, in the spectrum of HE 1448-0510 (shown here) and four other stars (for details see text).

to avoid underestimating the errors: since it is usually assumed that the individual results show a Gaussian error distribution as well, the width of the latter would be lower by a factor of $\sqrt{2}$ than the width we derived from our distribution of differences of the results of two exposures. However, we do not think that this standard assumption would be well justified here, since in many cases the differences are much larger than the typical statistical errors mentioned above.

Furthermore, when we take the mean value of two exposures, the errors are automatically reduced by a factor of $\sqrt{2}$, increasing the accuracy of the derived parameters. For stars with two or more spectra, those reduced Gaussian widths are $\Delta T_{\text {eff }}=360 \mathrm{~K}$ and $\Delta \log (g)=0.05 \mathrm{dex}$, which we regard as our best error estimates. More details are given in Table 2 .

An uncertainty is caused by the unknown metallicity of the program stars. We used solar metallicity, fully line-blanketed LTE models (see Sect. 3.1). A comparison shows that adopting a much lower metallicity $([\mathrm{m} / \mathrm{H}]=-2)$ in the LTE models has a negligible effect at $T_{\text {eff }}=32000 \mathrm{~K}$ (see also Heber et al. 2000 ) but increases $T_{\text {eff }}$ by up to $800 \mathrm{~K}$ at lower temperatures. Surface gravity is only marginally affected $(0.02 \mathrm{dex})$ while the helium abundance remains almost unchanged. Spectroscopic analyses of high resolution optical spectra of about two dozen
Table 2. Errors derived from the Gaussians in Fig. 5. For stars with one exposure (last column), the errors equal the Gaussian widths. For objects with two exposures or more, those values are divided by $\sqrt{2}$ (middle column).

\begin{tabular}{lll}
\hline \hline Quantity & Errors & Errors $^{a}$ \\
\hline$T_{\text {eff }}$ & $374 \mathrm{~K}$ & $529 \mathrm{~K}$ \\
$\log (g)$ & $0.049 \mathrm{dex}$ & $0.069 \mathrm{dex}$ \\
$\log (y)$ & $0.044 \mathrm{dex}$ & $0.062 \mathrm{dex}$ \\
$\log (L)^{b}$ & $0.038 \mathrm{dex}$ & $0.053 \mathrm{dex}$ \\
\hline
\end{tabular}

a For stars with only one useful exposure.

${ }^{b}$ Luminosity $L$.
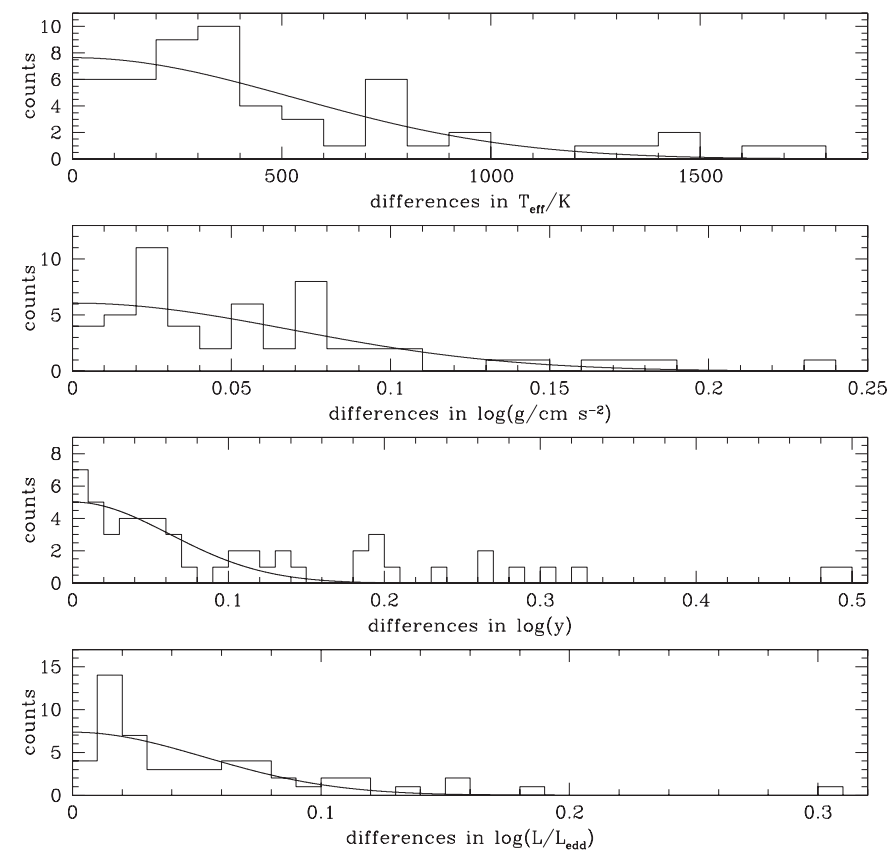

Fig. 5. Distributions of differences in atmospheric parameters between two exposures of non-composite stars. Solid lines show the Gaussians fitted to the data. From top to bottom the distributions for $T_{\text {eff }}, \log (g)$, $\log (y)$ and luminosity $L$ with respect to the Eddington luminosity are shown. 


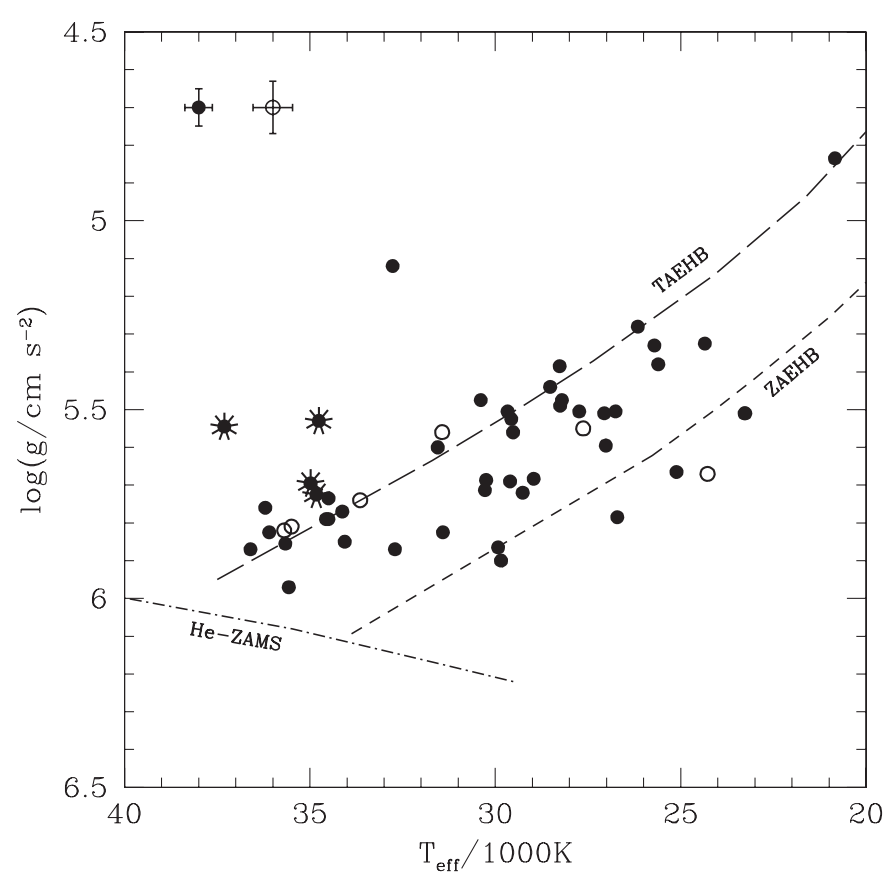

Fig. 6. Distribution of our sdB sample in the $T_{\text {eff }}-\log (g)$-plane. Results derived from only one exposure are shown as open circles, whereas filled circles represent mean values from two or more spectra. Errors are quoted separately for the former and the latter. Asterisks represent the four stars with peculiar $\mathrm{H} \alpha$ profiles. The ZAEHB (shortdashed line), TAEHB (long-dashed line) and He-ZAMS (dasheddotted line) are shown (see text for details).

bright sdB stars found near-solar iron abundances (Heber et al. 2000; Heber \& Edelmann 2004), corroborating our choice of metallicity. Hence the uncertainties introduced by the unknown metallicity are smaller than the errors of the spectroscopic analyses quoted above for the majority of our objects.

Another source of systematic error lies with the usage of both LTE and NLTE model atmospheres. Heber \& Edelmann (2004) have shown that a systematic offset in gravity exists: analysis with our grid of NLTE models leads to a gravity lower by 0.06 dex than derived from our LTE models, while the resulting effective temperatures and helium abundances are the same.

We find that neither of the effects above affects any of our conclusions (see Sect. 6).

\subsection{Effective temperature and surface gravity}

The fit results are listed in Table 1 . They were calculated as the mean value of the results from individual exposures of each star, using only spectra with $S / N \geq 10$. For nine stars, only one (useful) exposure was available (see Table 1).

Figure 6 shows the position of our sdB stars in the $T_{\text {eff }}-\log (g)$-plane, along with the ZAEHB and TAEHB for solar metallicity (Dorman et al. 1993) and the theoretical zeroage main sequence for pure helium stars (He-ZAMS, Paczyński 1971). Up to effective temperatures of $T_{\text {eff }} \approx 32000 \mathrm{~K}$, most of our sdB stars lie in the so-called EHB strip, defined by ZAEHB and TAEHB. For higher $T_{\text {eff }}$, almost all of the stars lie near the

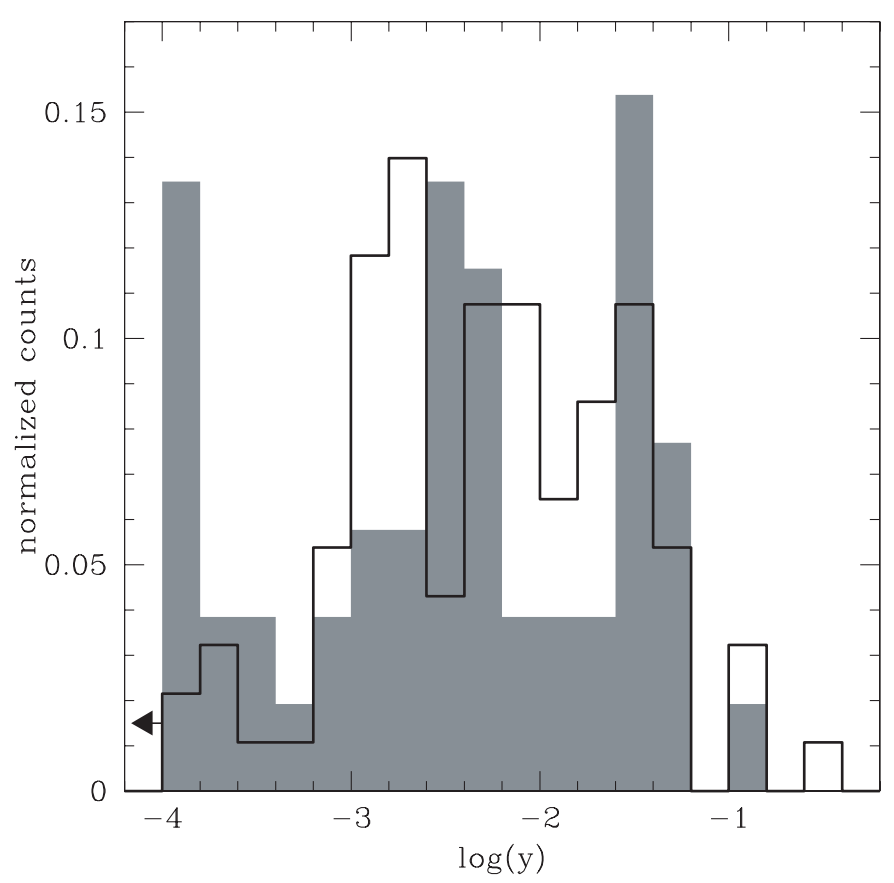

Fig. 7. Shaded histogram: distribution of photospheric helium abundance of our sdB stars. Open histogram: distribution from Edelmann et al. (2003). The value -4 is the lowest helium abundance in the model atmospheres of both studies. Since no extrapolation was done, it has to be regarded as an upper limit for the true abundance.

TAEHB or above it, including the four objects with peculiar $\mathrm{H} \alpha$ profiles. Six stars fall slightly below the ZAEHB. Possible explanations could be selection effects and/or evolutionary effects, which will be examined in Sects. 4.5 and 6, respectively.

From $T_{\text {eff }}$ and $\log (g)$, the luminosity could be calculated when a mass for the $\mathrm{sdB}$ is assumed. However, we prefer to use luminosity in units of the Eddington luminosity, thereby eliminating the mass. It is calculated from

$\log \left(\frac{L}{L_{\text {edd }}}\right)=4 \times \log \left(T_{\text {eff }} / \mathrm{K}\right)-\log \left(g / \mathrm{cm} \mathrm{s}^{-2}\right)-15.118$,

where pure electron scattering in a fully ionized hydrogen atmosphere is assumed.

In order to estimate distances we derive the absolute visual magnitude $M_{V}$ from the surface size and flux. To calculate the former, we need the surface gravity, and an assumption for the mass, taken to be $M_{\mathrm{sdB}}=0.5 M_{\odot}$. The surface flux is calculated from Kurucz model atmospheres (Napiwotzki 2001). Subsequently, the distance is derived from $M_{V}$ and the apparent visual brightness $M_{V}$. For most stars, though, only $m_{B}$ is available. Therefore, we adopted $B-V=-0.25$ for these objects, which is the typical value for non-composite sdB stars (Stark $\&$ Wade 2003, see also Fig. 2). We assumed Strömgren-y to be identical to $V$. All these additional quantities are given in Table 1.

\subsection{Helium abundance}

Our distribution of helium abundances (Fig. 7) shows a wide spread from the lower boundary of our model grids, 


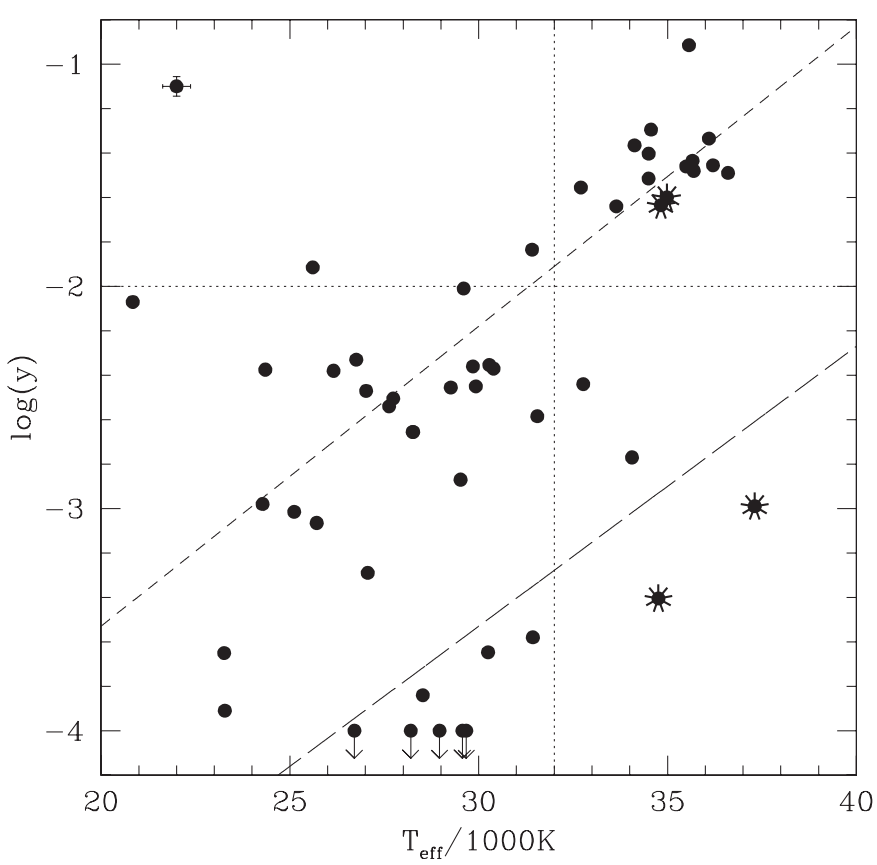

Fig. 8. Helium abundance vs. effective temperature. Asterisks represent the four stars with peculiar $\mathrm{H} \alpha$ profiles. Arrows denote upper limits for helium abundance. The dotted horizontal line marks a value of $\log (y)=-2$, the vertical line $T_{\text {eff }}=32000 \mathrm{~K}$. The dashed lines are from Edelmann et al. (2003), see text for details.

$\log (y)=-4$, with sometimes no helium visible at all, to slightly supersolar helium abundance $\log (y)=-0.92$. There is overall agreement with the results from the sample of Edelmann et al. (2003) (see Fig. 7), but we find a higher fraction of stars with very low helium abundances. Possible correlations with other quantities are presented in Figs. 8 and 9, where helium abundance is compared to effective temperature and luminosity, respectively. The comparison with $T_{\text {eff }}$ shows that for $\log (y)>-2$, almost all of the $\mathrm{sdB}$ stars are hotter than $T_{\text {eff }}>32000 \mathrm{~K}$ (see the dotted lines in Fig. 8). At these high temperatures, only two objects have a very low helium abundance $\log (y)<-3.0$, whereas there are twelve such stars at lower temperatures. Hence, at $T_{\text {eff }} \approx 32000 \mathrm{~K}$, our objects could be divided into two groups that are roughly correlated with spectral type sdB and sdOB, respectively, the latter having higher temperatures and an overall higher helium abundance.

In addition, a slight trend with luminosity can also be observed: at higher $T_{\text {eff }}$, the sdB stars are somewhat more luminous (see Table 1). Consequently, the average luminosity is higher for higher helium abundances, as seen in Fig. 9.

Interestingly, the two stars which have the lowest helium abundance of the high-temperature objects show peculiar $\mathrm{H} \alpha$ profiles, and are more luminous than the other two stars of this kind. One might thus speculate about underlying physical explanations. However, the $\mathrm{H} \alpha$ profiles of these two subgroups do not show any obvious differences, and from a statistical point of view there would be no significance in such a separation.

Edelmann et al. (2003) found two distinct sequences of sdB stars, separated mainly by an offset in helium abundance. Their corresponding linear fits are also plotted in Fig. 8.

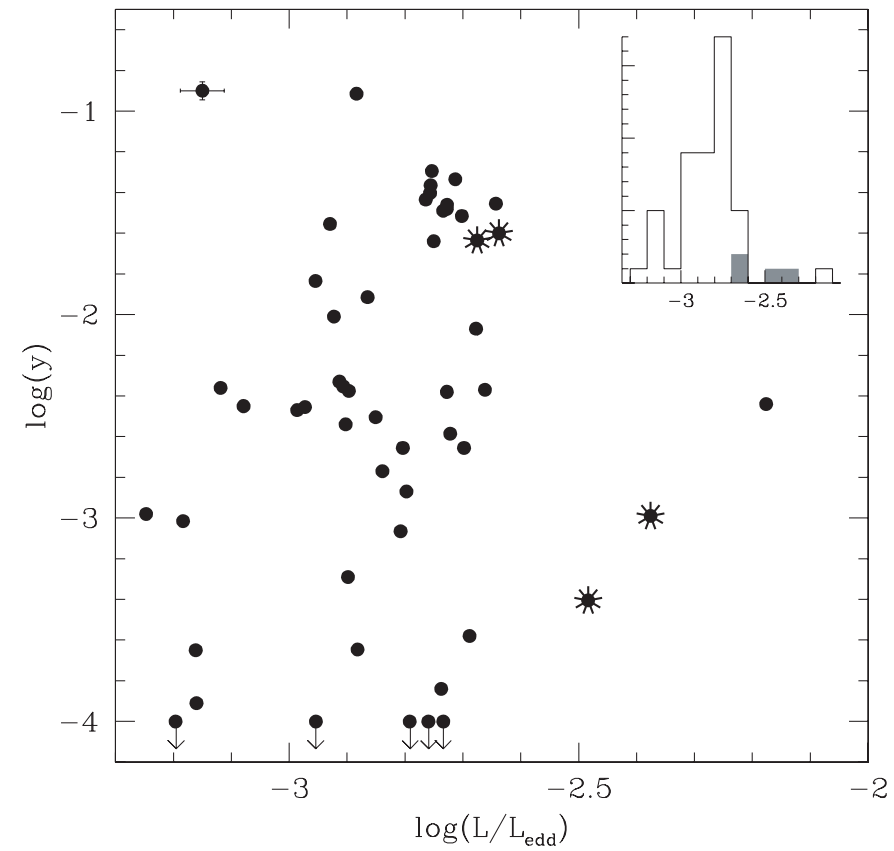

Fig. 9. Helium abundance vs. luminosity with respect to the Eddington luminosity. Asterisks represent the four stars with peculiar $\mathrm{H} \alpha$ profiles. Arrows denote upper limits for helium abundance. The inset shows the luminosity distribution as histogram for the four stars with peculiar $\mathrm{H} \alpha$ profiles (shaded) and the other objects (open).

The sequence for higher helium abundance (short dashed line) matches the majority of our objects quite well, especially at higher temperatures. The sequence for lower $\log (y)$ (long dashed line), with which Edelmann et al. (2003) matched about $1 / 6$ th of their data, can be neither confirmed nor rejected, mainly because we can only derive upper limits for five stars.

As discussed in previous work (e.g. Fontaine \& Chayer 1997), the diffusive equilibrium abundances of gravitational settling versus radiative levitation lie two orders of magnitude below the observed average helium abundances; hence, this diffusion model is too simplistic. Fontaine \& Chayer (1997) included mass loss of $10^{-14} M_{\odot} / \mathrm{yr}$ as an additional force in their diffusion models, and concluded that it is possible to reach equilibrium abundances as high as $\log (y)=-3$ after $t=10^{7} \mathrm{yr}$ on the EHB. Unglaub \& Bues (2001) showed that mass loss rates of $10^{-13} M_{\odot} / \mathrm{yr}$ can produce even higher helium abundances $\log (y)=-2$, again after $t=10^{7} \mathrm{yr}$. The large number of stars with $\log (y)>-2$ in our sample indicates that either the winds must be even stronger for reproducing the whole range of abundances, or alternative explanations have to be found.

Since the stellar winds of sdB stars are probably radiation driven, their mass loss rates are expected to be correlated with their luminosities (Pauldrach et al. 1988). However, it would be premature to use that correlation for interpreting our observed slight trend of helium abundance with luminosity. The metallicity plays an important role both for radiative levitation and the radiation-driven wind. It would therefore be of enormous interest to determine metallicities for our high-resolution sdB spectra and use them as input for theoretical calculations of radiative forces and wind mass loss, which, however, is beyond the scope of this paper. 


\subsection{Peculiar Ho profiles}

All four stars with peculiar $\mathrm{H} \alpha$ profiles have a higher luminosity than the majority of sdB stars (see inset in Fig. 9). This gives us a hint to a possible explanation: Heber et al. (2003) observed similar $\mathrm{H} \alpha$ profiles in the four most luminous objects of their sample, and suggested stellar winds as the cause. Similar to our data, their profile shapes show broadening around the core region, while the line depth is lower than in the model spectrum. The core itself is flattened in one case, and shows a faint emission in the other cases, consistent with our own observations.

First calculations of $\mathrm{H} \alpha$ lines from spherical NLTE models including mass loss have recently become available (Vink 2004). While they can explain the occurrence of small emissions in the core and, therefore, a weakening of the core, they are unable to match the observed widths of the line wings. In the latter respect, there is no improvement yet compared to the static, plane parallel NLTE models we use. Therefore the physical effects causing the extraordinary line broadening remain obscure. Obviously, there is a need for more sophisticated model atmospheres.

In the sdB mass loss calculations mentioned above, it is possible to reproduce the emission with mass loss rates of $10^{-11} M_{\odot} /$ yr. On the one hand, such a strong mass loss agrees with our conclusion from the previous subsection that mass loss rates above $10^{-13} M_{\odot} /$ yr would be needed to explain the upper part of the helium abundance range of sdBs. On the other hand, the helium abundance of the four stars discussed here lies between $-3.41 \leq \log (y) \leq-1.60$, leading us to recall the statement that stellar winds need not automatically cause a correlation of luminosity and helium abundance.

\subsection{Comparison with other samples}

$\mathrm{SdB}$ samples of comparable size were presented in the last decade by Saffer et al. (1994), Maxted et al. (2001) and Edelmann et al. (2003). Their main selection effect is the magnitude-limitation of the surveys that provided the targets (Palomar-Green Survey, HQS). In contrast, the selection effects of our sample are not well known. Since the SPY targets were drawn from various catalogs with criteria for selecting potential white dwarfs, the hot subdwarfs were included only by misclassification, rendering a definition of $\mathrm{sdB}$ selection criteria impossible. We now attempt to investigate whether the selection effects of our sample are similar to those of the samples just mentioned, or whether any systematic differences can be found.

An adequate means for comparing different samples of $\mathrm{sdB}$ stars is their respective cumulative luminosity function, shown in Fig. 10 for our sample along with the three studies named above. It can be seen that the SPY data agree quite well with the observations from Maxted et al. (2001) and Edelmann et al. (2003), whereas there is a larger average offset to the data of Saffer et al. (1994), which has already been discussed in Edelmann et al. (2003). Furthermore, several of our stars lie below the ZAEHB, which is plotted in Fig. 10 for various metallicities. Interestingly, the percentage of stars below the solar metallicity ZAEHB (12\%) is in better agreement with the

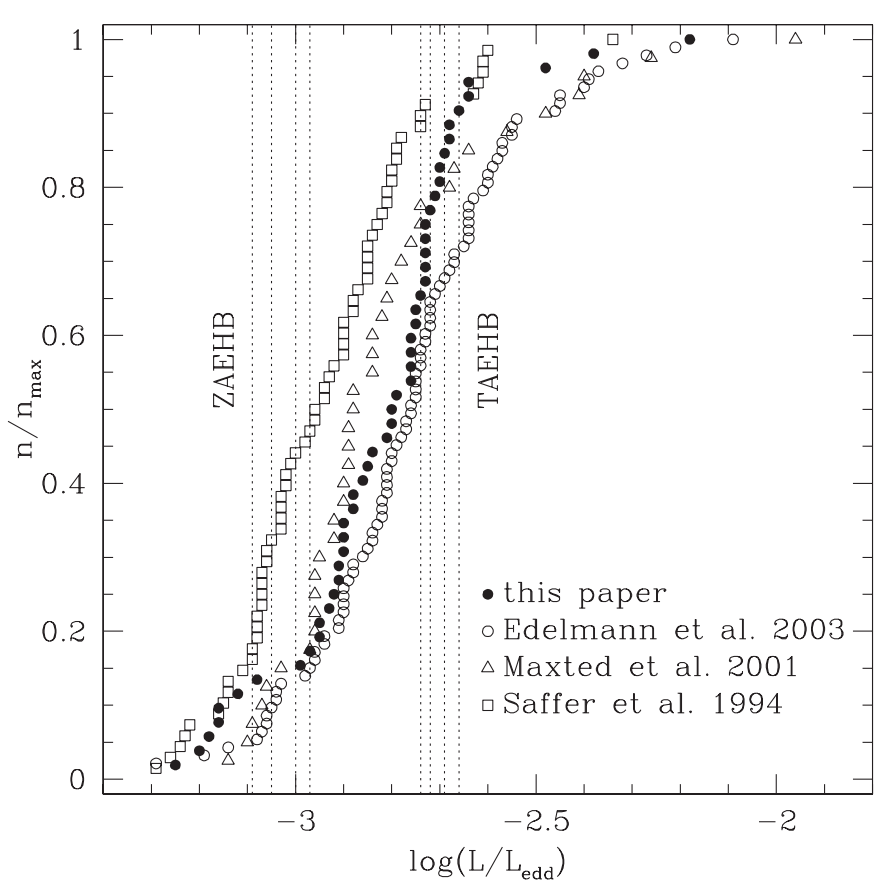

Fig. 10. Cumulative luminosity function: cumulative normalized counts versus luminosity in units of the Eddington luminosity. Shown are our data (filled circles) along with the measurements of Edelmann et al. (2003, open circles), Maxted et al. (2001, open triangles), and Saffer et al. (1994, open squares). ZAEHB and TAEHB are from Dorman et al. (1993) for metallicities $[\mathrm{Fe} / \mathrm{H}]=$ $0.00,-0.47,-1.48,-2.26$ from left to right.

observations from Saffer et al. (1994, 15\%) - despite the overall offset between the two functions - than with the other two studies (3\% and 5\%). Similarly, at the highest luminosities, our data again agree best with the sample of Saffer et al. (1994).

The observed moderate differences between the samples are most probably the result of differences in resolution, signalto-noise ratio, homogeneity, and models used for line profile fitting. As far as the latter are concerned, one must not only distinguish between the actual use of LTE or NLTE models (Saffer et al. 1994 only use the former), but also between the parameter regions in which they are applied: Edelmann et al. (2003) and Maxted et al. (2001) use NLTE models for objects having $T_{\text {eff }}>27000 \mathrm{~K}$, our study for sdBs with $T_{\text {eff }}>32000 \mathrm{~K}$. As another example of model differences, we mention that the LTE models of Saffer et al. (1994) did not include metal lineblanketing, while our LTE models do. Differences between NLTE and LTE model atmosphere results may be as large as $\Delta \log (L)=0.1$ dex (see Heber \& Edelmann 2004).

We conclude from the overall comparison of the samples and the lack of excessive deviations that the selection effects of our study are mainly similar to those of previous studies however, as far as flux limitation is concerned, we attempt to make a more reliable statement below. A more detailed discussion in the context of stellar evolution is deferred to Sect. 6 . 


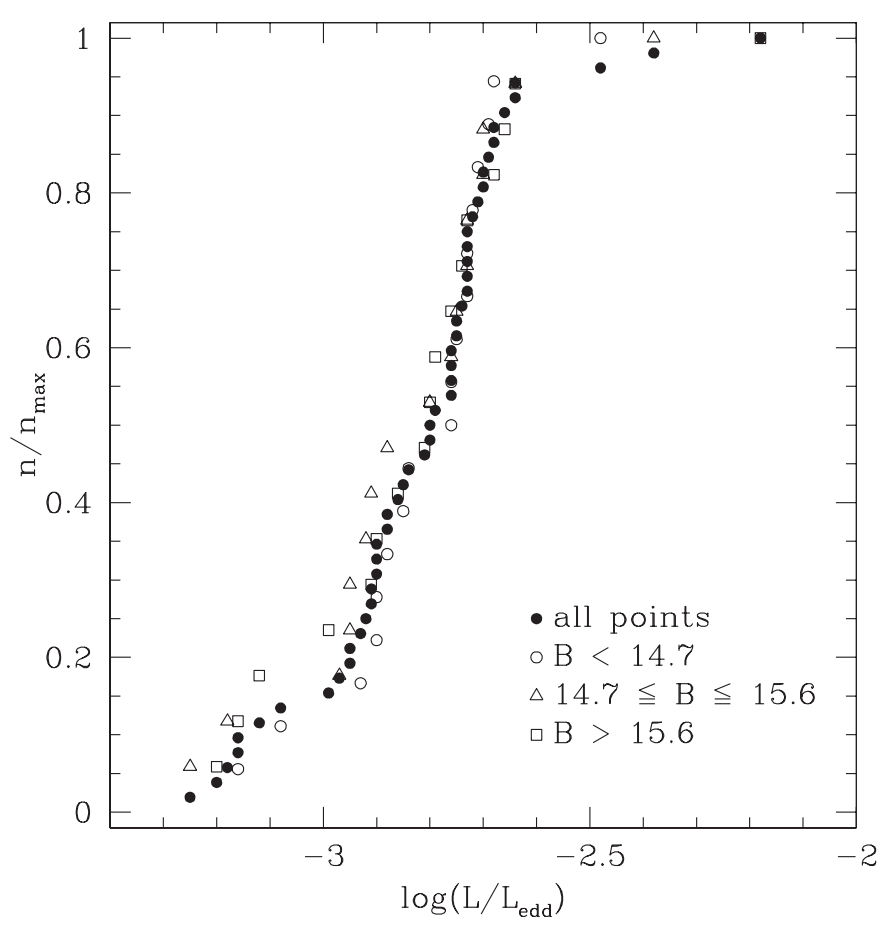

Fig. 11. Cumulative luminosity function for all our objects (filled circles, as in Fig. 10), as well as for three magnitude-selected subsets of equal number of stars: $B<14.7$ (open circles), $14.7 \leq B \leq 15.6$ (open triangles), $B>15.6$ (open squares).

\subsection{Flux limitation}

Our programme stars are drawn from flux-limited samples. This may introduce an observational bias against objects of lower luminosity, although the limiting magnitudes of the surveys (e.g. HES and HQS) are considerably deeper than the limit for SPY. If such a bias were present it would be more difficult to reconcile the lower end of the cumulative luminosity function with evolutionary predictions, since we would have to correct for low luminosity stars missing in our sample.

We therefore carried out a test by comparing the cumulative luminosity functions for three subsets of equal number of stars to that of the full sample (see Fig. 11). A bright subset ( $B<14.7)$, a medium bright subset $(14.7 \leq B \leq 15.6)$, and a faint subset $(B>15.6)$ were defined. As can be seen from Fig. 11, the cumulative luminosity function of the faint subset agrees very well with that of the bright and medium bright subsample, and all three agree well with that of the entire sample. We therefore conclude that no bias due to flux limitation is present. This makes the cumulative luminosity function a strong tool for the comparison of observation with predictions by the theory of stellar evolution (see Sect. 6).

\section{Double-lined objects}

\subsection{Companion classification}

For the $24 \mathrm{sdB}$ stars showing spectral signatures of a cool companion, accurate values of the stellar parameters cannot be expected from a simple model spectrum fit. In particular, the surface gravity is probably underestimated in many cases.
Nevertheless, we performed line profile fits of the lines $\mathrm{H} \delta$ and bluewards for 20 objects, attempting to minimize the effects of companion contribution, since the latter decreases with decreasing wavelength. In order to estimate the helium abundance, we had to include He I $4471 \AA$ in some cases, when no other helium line was present in the blue part of the spectrum. Our goal was to examine if the fit results can still be useful to determine absolute visual brightnesses $M_{V \mathrm{sdB}}$ of the sdBs, from which we can estimate the companion spectral type using $V$ and $J$ of the $\mathrm{sdB}+$ main sequence system.

For testing purposes, we "contaminated" three of our sdB stars showing no companion signatures with a certain amount of light from a cool companion. Since the SPY stars have not been flux calibrated, it was first necessary to create a typical spectral energy distribution for the sample stars. This was achieved by using flux calibrated low resolution spectra of $\mathrm{sdB}$ stars from Salomon (2003), which were chosen to have $T_{\text {eff- }}{ }^{-}$ and $\log (g)$-values similar to the sample stars. All our spectra were convolved with a Gaussian of $2.5 \AA F W H M$ and then rebinned to $1.0 \AA$ in order to match the spectra used for the companions (see below). After dividing the sample stars by the corresponding flux calibrated ones, a fit to the continuum of the resulting spectrum was performed manually. Finally, each sample star spectrum was divided by the respective fit curve, resulting in a pseudo-flux calibration for them.

For typical main sequence stars, we used spectra from the STELIB $^{2}$ database (Le Borgne et al. 2003) for companion types F9, G4, K0, and K2. Their resolution is $\Delta \lambda \leq 3 \AA$, which matches the treatment of our spectra described above. From the absolute visual brightness of the sdB stars and the chosen companion spectrum, flux contribution and total magnitudes in $B, V$, and $J$ were calculated (Bessell \& Brett 1988; Aller et al. 1982; Cox 2000), and composite spectra were produced (see Fig. 12). Now, Balmer line profile fitting for $\mathrm{H} \delta$ and bluewards was performed, keeping the helium abundance fixed for simplification. $M_{V \text { sdB }}$ was then calculated from the derived values as described in Sect. 4.2. Those values show that the effect of companion contribution can be quite large for F-type $(\Delta \log (g)=-0.23$ to $-0.6 \mathrm{dex})$ and G-type $(\Delta \log (g)=-0.06$ to $-0.29 \mathrm{dex})$ companions, which justifies our separate treatment of single-lined and double-lined sdB stars. For K-type companions the gravity estimate is almost unaffected $(\Delta \log (g)=-0.02$ to $-0.11 \mathrm{dex})$.

Let us now turn to the composite spectrum sdBs from SPY. We do not attempt to deconvolve the spectra, but analyze their photometric data $(B, J)$. For obtaining apparent brightnesses $m_{V \text { tot }}$ for the system and $m_{V \mathrm{sdB}}$ for the sdB alone, we begin with adopting $(B-V)_{\text {tot }}=-0.25^{3}$ to the system, the typical value for a single sdB star (Stark \& Wade 2003), and assume $m_{B \text { tot }}=m_{B \mathrm{sdB}}$. After having performed the calculation

\footnotetext{
${ }^{2}$ http://webast.ast.obs-mip.fr/stelib/

3 Although the Hamburg/ESO Survey provides $B-V$ colours (Christlieb et al. 2001), they have been calibrated only for main sequence stars with $B-V>-0^{\mathrm{m}} 1$ to an accuracy of \pm 0 . 1 . Since an even better accuracy would be necessary for our purposes, a calibration for sdB stars would have to be carried out beforehand, which is beyond the scope of this paper.
} 


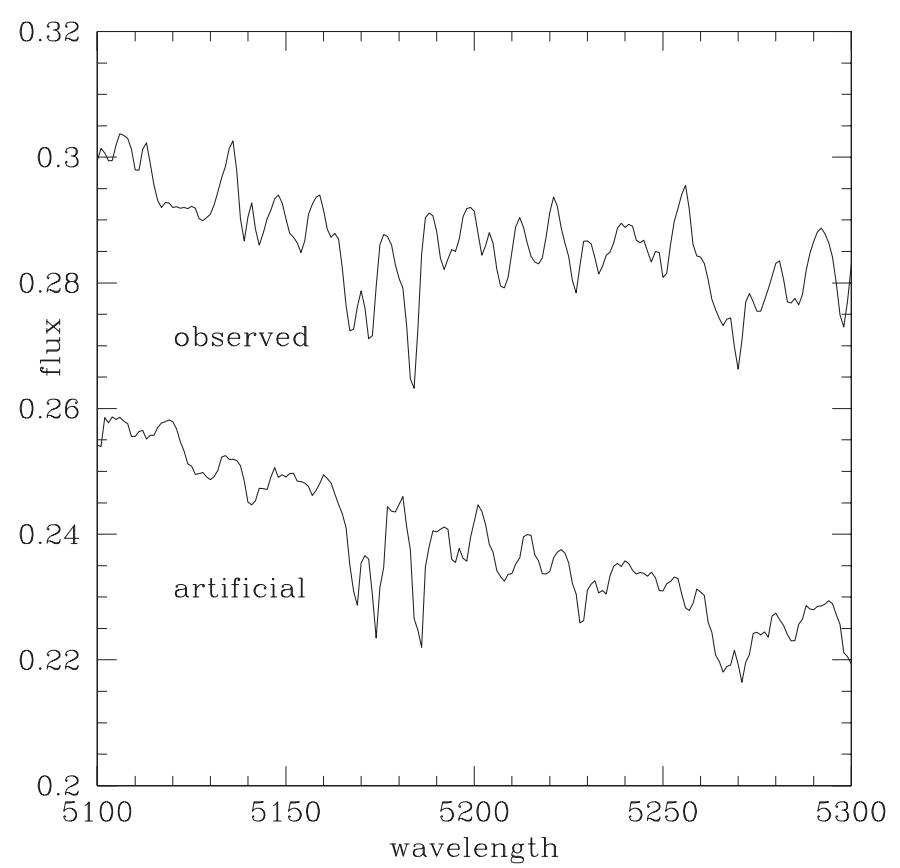

Fig. 12. Observed and artificial composite spectrum. Both spectra were scaled to the same flux level, and then displayed with an offset. Observed spectrum: HE 1309-1102, derived companion type G9, corrected $M_{V \mathrm{sdB}}=4$. 0 . Artificial spectrum: HE 2349-3135 with G4 companion, $M_{V \mathrm{sdB}}=3$. 86 .

of companion type once, as described below, we then correct $(B-V)_{\text {tot }}$ according to the calculated type and start over again with a new $m_{V \text { tot }}$.

The iteration itself is done in the following way: the distance modulus of the system is calculated from $M_{V \mathrm{sdB}}$ and $m_{V \text { sdB }}$. The $J$ magnitude is determined by subtracting the typical value of $V-J=-0.6$ for single sdB stars (Stark \& Wade 2003, consistent with our own findings). Then, by comparison with the measured $m_{J \text { tot }}, m_{J \text { comp }}$ is calculated, leading to $M_{J \text { comp }}$ when combined with the distance modulus. This automatically yields a companion type when compared to literature (Bessell \& Brett 1988; Aller et al. 1982; Cox 2000), providing us with a value for $M_{V \text { comp }}$ and subsequently $m_{V \text { comp }}$. Now, a more reliable $m_{V \mathrm{sdB}}$ was calculated from the latter and $m_{V \text { tot }}$, and the next iteration step was performed. The iteration is stopped when the spectral subtype of the companion stays the same from one step to the next, reflecting our maximum achievable accuracy.

The iteration leads to good estimates of the companion types for all our artificial composite sdB stars, even when the companion contribution to the total flux is quite high. The difference of input and output type lies between 0 and 2 subtypes. This clearly shows that the method can indeed be applied to our observed sample of composite sdB stars. However, we must point out three sources of error:

1. We adopt a typical value of $(V-J)_{\mathrm{sdB}}=-0 . \mathrm{m} 6$. Since the observed scatter is quite large (see Fig. 2 and Stark \& Wade 2003), this could produce errors in our companion types. The estimated error for $B$, from which $V$ is determined (see below), is $0.2, J$ errors from 2 MASS lie at about 0.1 . For
$\Delta(V-J)_{\mathrm{sdB}}= \pm 0.2$, the difference is two subtypes or less for low companion contribution (i.e. $\mathrm{K}$ types), and even smaller for brighter companion stars (i.e. earlier types).

2. Similarly, we adopt a typical value of $(B-V)_{\mathrm{sdB}}=-0.25$, which in reality also shows some small scatter of the order of $0^{\mathrm{m}} 1$ (Altmann et al. 2004).

3. For deriving companion types and absolute visual brightnesses, we use typical values given in the literature, again neglecting the natural scatter contained in the true values.

We therefore conclude that the error limits of our results are a few subtypes. We do not account for extinction because of the relatively high galactic latitudes of our stars. The nine objects which are also in the sample of Altmann et al. (2004, see Table 1) have $0.004 \leq E_{B-V} \leq 0.035$, justifying that approach. However, extinction might be important for PG $0258+184$ and PG 2122+157, where $E_{B-V} \geq 0.1$ (Schlegel et al. 1998). It follows that for these objects the $\mathrm{sdB}$ is somewhat brighter than what is derived by our iteration, and hence the companion is slightly fainter, and of later type.

Our derived companion types for the observed composite sdB stars are shown in Table 3. They range from F7, which is an early-type limit in our iteration, to K3 for the stars showing $\mathrm{Mg} \mathrm{I}$ in the spectrum. As expected, all of the five companions that were only deduced from a contribution at $\mathrm{H} \alpha$ are of relatively late spectral type (K1 to K7). The derived atmospheric parameters for the corresponding sdBs are not affected by companion spectral contribution, since the latter is negligible for the lines $\mathrm{H} \delta$ and bluewards, where line profile fitting was performed. All composite sdB stars will be investigated in more detail in the future, when we will attempt to deconvolve their spectra as described in Aznar Cuadrado \& Jeffery $(2001,2002)$.

\subsection{Main sequence or subgiant companions}

The luminosity class of cool companions to sdBs has been discussed extensively. For example, Allard et al. (1994) and Jeffery \& Pollacco (1998) claimed that the companions to their sdB stars are mostly overluminous as compared to main sequence stars. In contrast, Aznar Cuadrado \& Jeffery (2001, 2002) found their companion stars to be mostly main sequence objects. Recently, Stark \& Wade (2003) again discussed the nature of companions to $\mathrm{sdB}$ stars, pointing out that optical and infrared photometry alone is not able to distinguish between the main sequence and the subgiant scenario.

Figure 13 shows the absolute visual brightness distribution of our non-composite sdB stars. The values range from 3 . 6 to 5 . 0 , with two extreme values at 2.76 and 2.94 from one post-EHB and one potential HBB object, respectively. In the previous section, our iterative calculations of companion types yielded as a byproduct absolute magnitudes of the sdB component (see Table 3), which are also shown in Fig. 13. These values are in very good agreement with the ones derived for the non-composite sdB stars, and are mostly much fainter than the typical values of $M_{V} \approx 3$ for subgiant stars (Aller et al. 1982; also see Reid, http://www-int.stsci.edu/ inr/cmd.html, and 
Table 3. Determination of companion types, the results of which are listed in the second column. The first 15 objects show Mg I in the spectrum, the last five stars only show a continuum contribution at $\mathrm{H} \alpha$.

\begin{tabular}{lllllllll}
\hline \hline Object & Type & $\begin{array}{l}m_{B \text { tot }} \\
\mathrm{mag}\end{array}$ & $\begin{array}{l}m_{J \text { tot }}{ }^{2} \\
\mathrm{mag}\end{array}$ & $\begin{array}{l}m_{V \text { sdB }} \\
\mathrm{mag}\end{array}$ & $\begin{array}{l}m_{V \text { comp }} \\
\mathrm{mag}\end{array}$ & $\begin{array}{l}(B-V)_{\text {tot }} \\
\mathrm{mag}\end{array}$ & $\begin{array}{l}M_{V \text { sd }}^{\text {out }} \\
\mathrm{mag}\end{array}$ & Remark \\
\hline HE 1038-2326 & F7 & 15.9 & 15.22 & 16.7 & 16.4 & +0.11 & 4.0 & F7 or earlier \\
HE 1140-0500 & G1 & 14.8 & 15.17 & 15.1 & 17.3 & -0.18 & 2.4 & \\
HE 1221-2618 & G0 & 14.7 & 13.91 & 15.4 & 15.2 & +0.13 & 4.6 & \\
HE 1309-1102 & G9 & 16.1 & 16.14 & 16.4 & 18.2 & -0.13 & 4.0 & \\
HE 1352-1827 & G3 & 16.0 & 15.75 & 16.5 & 17.2 & -0.03 & 4.1 & \\
HE 1422-1851 & F9 & 16.3 & 16.17 & 16.7 & 17.7 & -0.07 & 3.2 & \\
HE 1441-0558 & G5 & 14.4 & 13.79 & 15.0 & 15.2 & +0.08 & 4.9 & \\
HE 2156-3927 & K3 & 14.26 & 14.44 & 14.5 & 17.2 & -0.18 & 4.1 & Altmann et al. $(2004):(B-V)_{\text {tot }}=-0.19$ \\
HE 2322-0617 & G9 & 15.7 & 15.58 & 16.1 & 17.4 & -0.09 & 4.3 & \\
HE 2322-4559 & G4 & 15.5 & 15.25 & 16.0 & 16.8 & -0.03 & 4.2 & \\
HS 1536+0944 & K0 & 15.6 & 15.27 & 16.0 & 17.2 & -0.07 & 4.7 & \\
HS 2216+1833 & G1 & 13.8 & 13.32 & 14.4 & 14.7 & +0.04 & 4.2 & \\
PG 0258+184 & G8 & 15.3 & 14.99 & 15.7 & 16.7 & -0.06 & 4.4 & F7 or earlier \\
PG 2122+157 & F7 & 15.0 & 13.95 & 16.4 & 15.0 & +0.28 & 5.1 & F7 or earlier \\
TON S 155 & F7 & $16.1 c$ & 15.69 & 16.8 & 17.0 & +0.02 & 3.5 & \\
\hline HE 1200-0931 & K1 & 16.2 & 16.07 & 16.5 & 18.1 & -0.11 & 4.6 & \\
HE 1254-1540 & K7 & 15.2 & 15.56 & 15.5 & 19.0 & -0.22 & 4.3 & \\
HE 1419-1205 & K5 & 16.2 & 16.49 & 16.5 & 19.6 & -0.21 & 4.2 & K4 or later \\
HS 2125+1105 & K4 & 16.4 & $\geq 16.5$ & 16.7 & 19.2 & -0.18 & 4.5 & \\
KUV 01542-0710 & K2 & $16.3{ }^{b}$ & 16.30 & 16.4 & 18.8 & -0.18 & 4.0 & \\
\hline
\end{tabular}

a Data from 2MASS, obtained by usage of the VizieR database (Ochsenbein et al. 2000).

${ }^{b}$ Johnson $V$ magnitude.

$c$ Strömgren $y$ magnitude.

references therein). This clearly proves that the majority of our companion stars are indeed main sequence stars.

In addition, our values of $M_{V \mathrm{sdB}}$ are supported by observations of twelve EHB stars in the globular cluster NGC 6752. Its known distance allows a direct determination of their absolute brightnesses, which lie in the range $3 \cdot 80 \leq M_{V \mathrm{sdB}} \leq$ $4 \mathrm{~m} 66$, with a median value of $M_{V \mathrm{sdB}}=4.21$ (Moehler et al. 1997). The median value for our non-composite objects is $M_{V \mathrm{sdB}}=4.20$, and $M_{V \mathrm{sdB}}=4.2$ for the sdB component of the composites.

We therefore conclude that the vast majority of cool companions to observed sdB stars are main sequence stars, and consider the question of companion luminosity class to be settled. However, it follows automatically that $\mathrm{sdB}+$ subgiant systems can hardly be detected because of the subgiant being brighter than the typical sdB. Since "our goal should be to describe Nature, not to confirm our own favorite prejudices" (R. A. Wade), it has to be pointed out that such systems may exist, yet mostly remain undetected.

\subsection{Helium abundance}

Aznar Cuadrado \& Jeffery (2002) find that the compositespectrum sdB stars show lower photospheric helium abundances than the non-composite objects, the former having $\log (y)<-2$. Our derived helium abundances for the

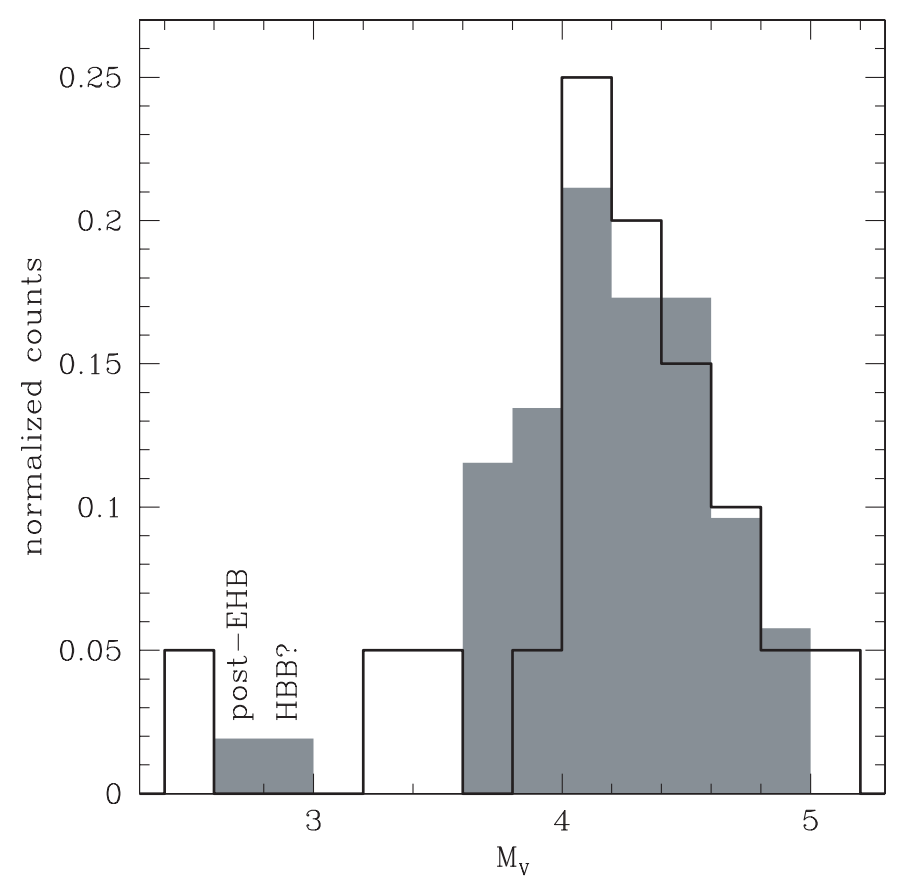

Fig. 13. Shaded histogram: distribution of absolute visual brightness of non-composite objects. The two leftmost counts come from the post-EHB star HE 0415-2417 and the potential HBB star HE 0151-3919. Open histogram: distribution of 20 out of $24 \mathrm{sdBs}$ with cool companions, as derived from the iteration described in Sect. 5.1. 


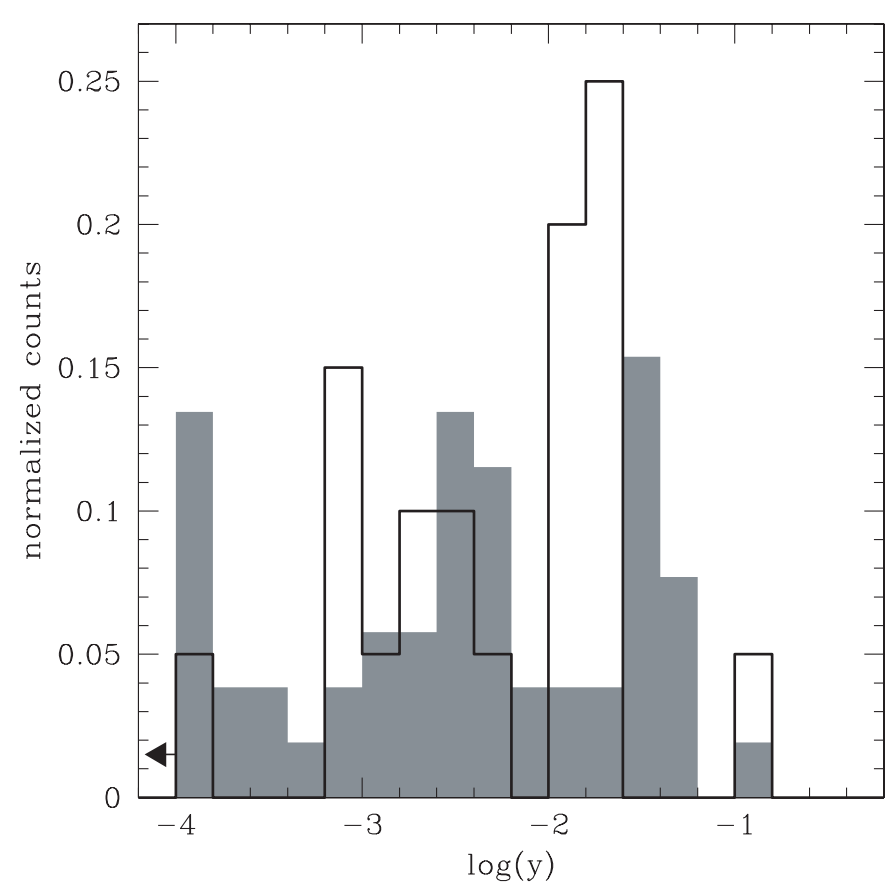

Fig. 14. Shaded histogram: distribution of photospheric helium abundance of non-composite sdB stars, as in Fig. 7. Open histogram: distribution of 20 out of 24 objects with cool companions.

20 composite sdB stars for which fitting was performed can be seen in Table 1. They are expected to be lower limits, since the presence of a cool companion contributing to the total flux dilutes the helium lines and therefore decreases the helium abundance derived from spectral fitting. A similar test as described in Sect. 5.1, using HE 2237+0150, shows that the helium abundance is underestimated by 0.17 dex for an F9 companion and 0.03 dex for a $\mathrm{K} 2$ companion.

Figure 14 contains the derived values as a histogram together with the results for non-composite sdB stars. It is clear that the helium abundances for composite-spectrum sdB stars cover the whole range of abundances from $\log (y) \leq-4$ to $\log (y)>-1$, with $50 \%$ of the stars having $\log (y)>-2$, in obvious disagreement with the observations of Aznar Cuadrado \& Jeffery (2002).

The stars discussed here have formed in binaries with relatively large orbital separations and long periods (1st RLOF channel, see Sect. 6). Aznar Cuadrado \& Jeffery (2002) suggested that the different strengths of tidal effects in shortperiod and long-period sdB binaries may cause differences in the atmospheric helium content of these two groups. This argument was taken up by Edelmann et al. (2003) as a possible explanation for their two distinct $\mathrm{sdB}$ sequences in the $T_{\text {eff }}-\log (y)$-plane (see Fig. 8). The absence of any differences between single-lined and double-lined sdBs in our sample rules out such a correlation. Furthermore, a large number of the noncomposite sdB stars may be single objects, which is not taken into consideration in these discussions.

\section{Observation versus theory}

In order to draw conclusions about potential formation scenarios and the evolutionary status of our objects, we consider two theoretical studies. Dorman et al. (1993) computed evolutionary tracks for the canonical core mass established by the helium flash in the core of a red giant star. The core mass depends on metallicity, since the elemental abundances determine the core mass necessary for helium ignition on the first giant branch (FGB). Dorman et al. (1993) used various metallicities and envelope masses, defining the standard ZAEHB and TAEHB, as well as EHB and post-EHB evolution, which we have already used in Sect. 4.5. We will take those calculations as representative for scenarios of single star sdB evolution, which we define in such a way that any companion star that might be present does not affect the evolution of the sdB.

In contrast, the binary population synthesis calculations of HPMM aim to distinguish between several binary formation channels of sdB stars and then simulate the evolution of the resulting subsamples. Those calculations are the most recent and most extensive simulations for binary formation scenarios of the $\mathrm{sdB}$ population. They can be divided into three channels:

(i) The common envelope ejection channel. Dynamically unstable mass transfer in a binary system with one star being on the FGB results in the formation of a CE. Subsequently, the spiraling-in of both stars and finally the ejection of the $\mathrm{CE}$ can lead to an sdB in a very close binary system, if the giant's core is still able to ignite helium. The second member of the system can either be a main sequence star ("1st $\mathrm{CE}$ ejection channel") or already a white dwarf ("2nd CE ejection channel").

(ii) The stable Roche lobe overflow channel. This channel is analogous to the CE ejection channel, except for the mass transfer being stable. The value of $q_{\text {crit }}$ decides which of the two channels applies to a system: only if the ratio of red giant mass to companion mass is below $q_{\text {crit }}$ (adopted to be either 1.2 or 1.5 in the simulations) is mass transfer stable. This results in binary systems with a much larger separation and therefore much longer periods, since there is no spiraling-in phase affecting the orbital parameters. Again, one has to distinguish between the "1st RLOF channel" and the "2nd RLOF channel". However, as shown in HPMM, the 2nd RLOF channel (stable mass transfer onto a white dwarf) is negligible because white dwarfs of sufficiently high masses are extremely rare.

(iii) The merger channel. The merger of two He-WDs caused by loss of energy due to gravitational wave radiation can produce an sdB star, if the mass is sufficient for core helium burning. Unlike sdB stars from other channels, the objects produced here are single stars.

To examine the effects of various poorly known physical parameters in the scenarios just described, HPMM produced twelve simulation sets for different values of the following quantities: CE ejection efficiency $\alpha_{\mathrm{CE}}$, fraction $\alpha_{\text {th }}$ of CE thermal energy used for its ejection, critical mass ratio $q_{\text {crit }}$ above which mass transfer is stable on the RGB, initial mass ratio distribution of the progenitor binary systems, and metallicity. 
HPMM compared the periods and minimum companion masses for the single-lined sdB binaries observed by Maxted et al. (2001) and Morales-Rueda et al. (2003) to the predictions of their numerical models and selected a simulation set that matches those observations best ("best-fit model"). However, this comparison does not include stars which formed via the merger channel, since these are single objects. Hence, it is very interesting to compare HPMM's predictions directly with our spectroscopic results, because our sample is not biased against single stars.

To make a comparison possible and reasonable, it is imperative to consider observational selection effects and apply them in the same way to the theoretical sample. HPMM showed that the so-called GK selection effect is the most important one, since observational studies typically select against G, K, and earlier type main sequence companions. Those either outshine the sdB or cause a composite spectrum which renders the analysis of the sdB difficult or even impossible. In the simulations, HPMM apply this effect by excluding all sdB+main sequence systems with the companion either being brighter than the sdB or having $T_{\text {eff }}>4000 \mathrm{~K}$, corresponding to $\mathrm{M}$ and very late K types. Since we excluded all composite spectrum stars carefully, our sample matches this approach, and allows a detailed comparison of our data with the theoretical predictions of HPMM.

\subsection{Single star evolution}

Figure 15 compares our results to several evolutionary tracks calculated by Dorman et al. (1993) for different core and envelope masses. It can be clearly seen that at least one of our stars (HE 0415-2417 at $\left.T_{\text {eff }}=32768 \mathrm{~K}, \log (g)=5.12\right)$ must be in the post-EHB stage of evolution (dotted part of the tracks in Fig. 15). For a more thorough comparison with these theoretical predictions, we plot in Fig. 16 our cumulative luminosity function from Sect. 4.5 along with the luminosity evolution with time given by the described tracks, until they reach an effective temperature of $40000 \mathrm{~K}$. This limit follows from our selection criteria. The function should reflect the "speed of evolution" across the EHB and in the post-EHB stage.

It has been argued previously that most stars would be expected to lie near the ZAEHB (e.g. Edelmann et al. 2003). The theoretical tracks, however, show a linear time-luminosityrelation while the star is in the EHB strip. The respective ZAEHB is defined by the starting point of each track, the TAEHB is clearly marked by the sharp bend in the tracks. We therefore have to expect a homogeneous distribution of $\mathrm{sdB}$ stars through the EHB strip, if there are no observational selection effects.

While the discussed evolutionary tracks lie at somewhat lower average luminosity, their slope matches our data well, except for the six stars at luminosities lower than any of the plotted tracks. As shown in Sect. 4.6, any potential effects of magnitude limitation are negligible, and do not affect this comparison. Furthermore, replacing NLTE models by metal lineblanketed LTE models for the hottest stars would primarily lower the luminosity mainly at the top part of the cumulative

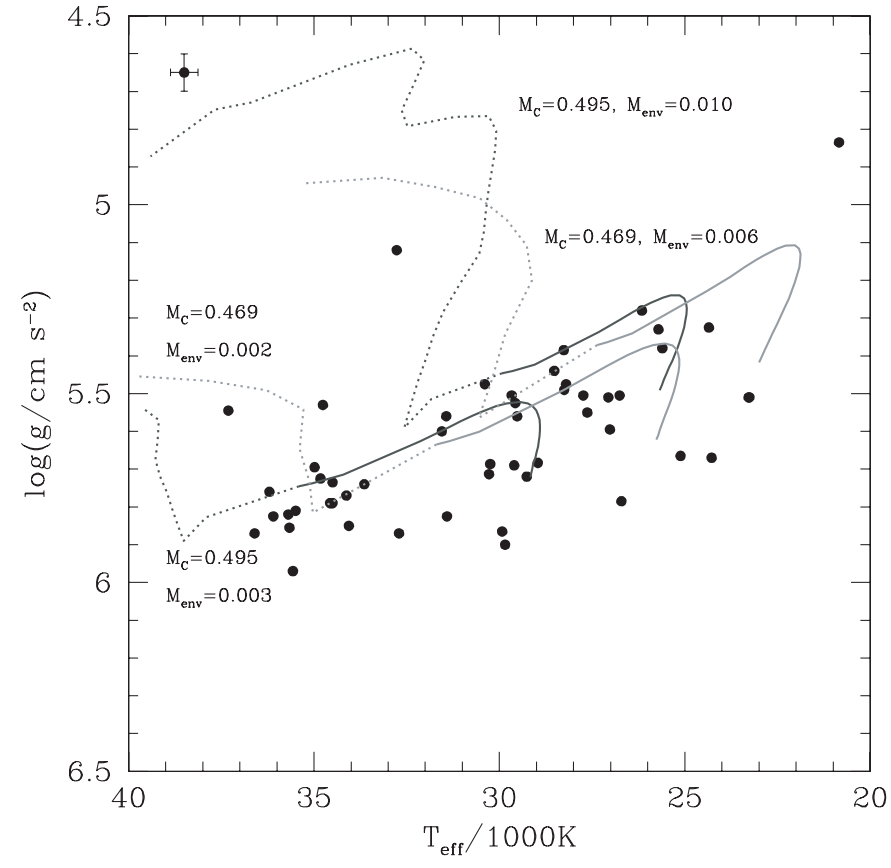

Fig. 15. $T_{\text {eff }}$ and $\log (g)$ values of our sdBs as shown in Fig. 6, along with evolutionary tracks calculated by Dorman et al. (1993). Tracks are shown for solar metallicity (helium core mass $M_{\text {core }}=0.469 M_{\odot}$, light grey lines $)$ as well as for $[\mathrm{Fe} / \mathrm{H}]=-2.26\left(M_{\text {core }}=0.495 M_{\odot}\right.$, dark grey lines). The respective envelope masses $M_{\text {env }}$ are displayed in the figure. The solid part of the tracks marks the core helium burning phase, while this has already ceased when the star is on the dotted part of the tracks.

luminosity function by about $0.06 \mathrm{dex}$. Adopting a very low metallicity would increase the luminosities by 0.03 dex only. Hence these systematic errors are unimportant (see Sect. 4.1).

\subsection{Close binary evolution}

We proceed with a detailed comparison of our measurements with all twelve simulation sets of HPMM. Our goal is to exclude several sets and their respective parameter configurations, to test HPMM's choice of the best-fit model, and to check whether these calculations can well reproduce the observed sdB distribution.

A short overview of the input parameters that characterize the simulation sets is given now. Three sets in a row $(1+2+3$, $4+5+6$, etc.) constitute a sequence in both the common envelope ejection efficiency $\alpha_{\mathrm{CE}}$ and the thermal energy fraction $\alpha_{\text {th }}$ used for ejection. The first set always has $\alpha_{\mathrm{CE}}=\alpha_{\text {th }}=0.5$, the second $\alpha_{\mathrm{CE}}=\alpha_{\mathrm{th}}=0.75$, the third $\alpha_{\mathrm{CE}}=\alpha_{\mathrm{th}}=1.0$. Sets 1 to 6 have a critical mass ratio $q_{\text {crit }}=1.5$, whereas $q_{\text {crit }}=1.2$ is used for sets 7 to 12 , allowing stable mass transfer only for a narrower mass range than the former value. For sets 4,5 , and 6 , an uncorrelated mass distribution had been adopted for the stars in $\mathrm{sdB}$ progenitor binaries, whereas in all other sets, HPMM used a constant distribution of the progenitor system mass ratio. Sets 10,11 , and 12 were calculated with a typical thick disk metallicity $(Z=0.004)$ while the other sets are for population I metallicity $(Z=0.02)$. 


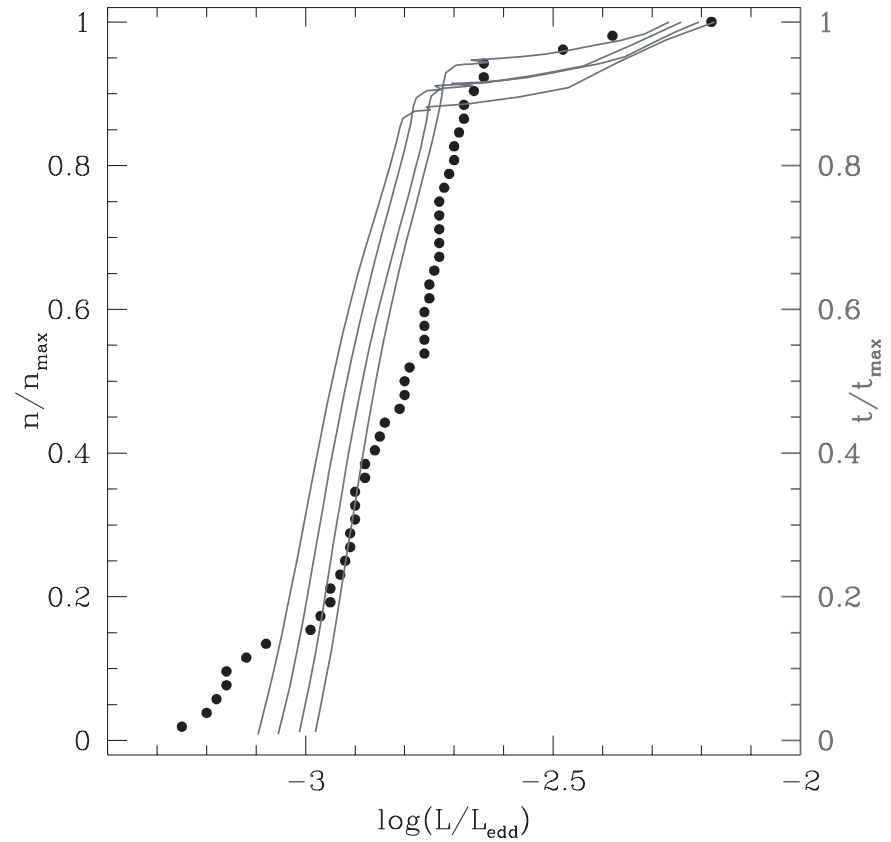

Fig. 16. Cumulative luminosity function of our sdB stars as shown in Fig. 10, along with sdB luminosity evolution with time as calculated by Dorman et al. (1993). The evolutionary tracks (grey lines) are followed from the ZAEHB until they leave our $T_{\text {eff }}-\log (g)$-parameter space $\left(T_{\text {eff }}<40000 \mathrm{~K}, \log (g)>4.5\right)$. For these tracks, the metallicities are from left to right: $[\mathrm{Fe} / \mathrm{H}]=0.00,-0.47,-1.48,-2.26$ (corresponding to core masses of $\left.M_{\text {core }}=0.469,0.475,0.485,0.495 M_{\odot}\right)$. For the leftmost track the envelope mass is $M_{\text {env }}=0.002 M_{\odot}$, for the other tracks $M_{\text {env }}=0.003 M_{\odot}$.

\subsubsection{The $T_{\text {eff }}-\log (g)$-plane: Visual inspection}

Figure 17 shows the simulated data from HPMM in the $T_{\text {eff }}-\log (g)$-plane. The number density is grey-scale coded; higher densities of sdB stars correspond to darker grey shading. Note that the total number of simulated stars is not the same for different simulation sets. Overlaid on each simulation data set are our measurements. The simulations shown here include the GK selection effect, as explained above, and are therefore well suited for comparison with our observations.

Sets 4,5 , and 6 do not match our data, since their predicted $\mathrm{sdB}$ density at higher temperatures is much lower than for cooler stars, in obvious contrast to our measurements. Sets 3, 9, and 12 all show a significant number of objects at low $T_{\text {eff }}$ and higher $\log (g)$, i.e. in the lower right area of the diagram. These stars have somewhat lower luminosities, and are not seen in our data. Since no bias due to flux limitation is present in our sample (see Sect. 4.6), we can state a trend against these simulation sets.

From visual judgement only, sets 1, 2, 7, 8, 10, and 11 all match the observed sdB distribution well. In all sets, a trend can be seen that the average surface gravity at lower temperatures is somewhat higher in the simulations than in our data. Again, this cannot be explained with a potential flux limitation bias, therefore, the observed shift could point towards the necessity of refining some model details.

Two stars are not matched by any simulation set: $\mathrm{HE} 0151-3919$ at $T_{\text {eff }}=20841 \mathrm{~K}, \log (g)=4.83$ and
HE $0415-2417$ at $T_{\text {eff }}=32768 \mathrm{~K}, \log (g)=5.12$. We cannot rule out that the former is a blue HB star, which will ascend the AGB after core helium exhaustion and therefore may belong to a different evolutionary group than the EHB stars. The latter is believed to be in the post-EHB stage (see Fig. 15), which lasts only a fraction of the time spent on the EHB. Since there are no additional stars observed in this parameter region, we do not consider this to be a discrepancy with theory.

In Sect. 4.3 we found that at higher $T_{\text {eff }}$ the sdB stars are somewhat more luminous, and tend to have a higher helium abundance. The corresponding $T_{\text {eff }}-\log (g)$-range of simulated data points is dominated by stars that formed in the merger channel. Since most of the hydrogen is expected to be burned up during the merging process, those objects are more likely to have a higher helium abundance than sdB stars from other channels (Han et al. 2002). This qualitative agreement with our results can be tested in a quantitative way by using radial velocity variations to distinguish between single objects from the merger channel and binaries (Napiwotzki et al. 2004; Napiwotzki et al. in prep.).

\subsubsection{The $T_{\text {eff }}-\log (g)$ plane: Statistical analysis}

To check the visual judgement, we performed a statistical test for the match of the different sets, using the maximum likelihood method. Thereby, we adopt as probability for each of our sdBs the fraction of simulated stars in a box around the data point, and then multiply the probabilities of all points. The size of the box corresponds to two times our errors for stars with one exposure, which was carefully chosen to avoid random fluctuations on the one hand, and too much smoothing on the other hand. In Fig. 17 the bin size is only half of that value - i.e. it equals the errors - and one can clearly see that quite large fluctuations can occur, since the total number of simulated stars only lies between 1000 and 2000, and is distributed over several hundred bins.

The ranking derived from the likelihood of each set to produce the observed distribution is given in Table 4, and clearly favors set 10 , which is characterized by a low efficiency $\left(\alpha_{\mathrm{CE}}=\alpha_{\mathrm{th}}=0.5\right)$, low metallicity $(Z=0.004)$, and a constant mass ratio of the progenitor binaries. We want to point out that the given likelihood ratios cannot be exact, since the simulation data are provided as discrete number counts rather than a smooth distribution function. Nevertheless, the overall ranking can be considered reliable. Sets 8,11 , and 2 form a group that still matches the observations well, while all of the following sets give a poor match to our data. Note that HPMM favored sets 2 and 8, where the former is their best-fit model. The rejection of sets 4,5 , and 6 is nicely confirmed, since they rank last, together with set 9 . Similarly, the trend that we stated above against sets 3,9 , and 12 is supported by the statistical analysis.

\subsubsection{The cumulative luminosity function}

Figure 18 compares the cumulative luminosity function from all simulation sets of HPMM with our data. It is obvious that 


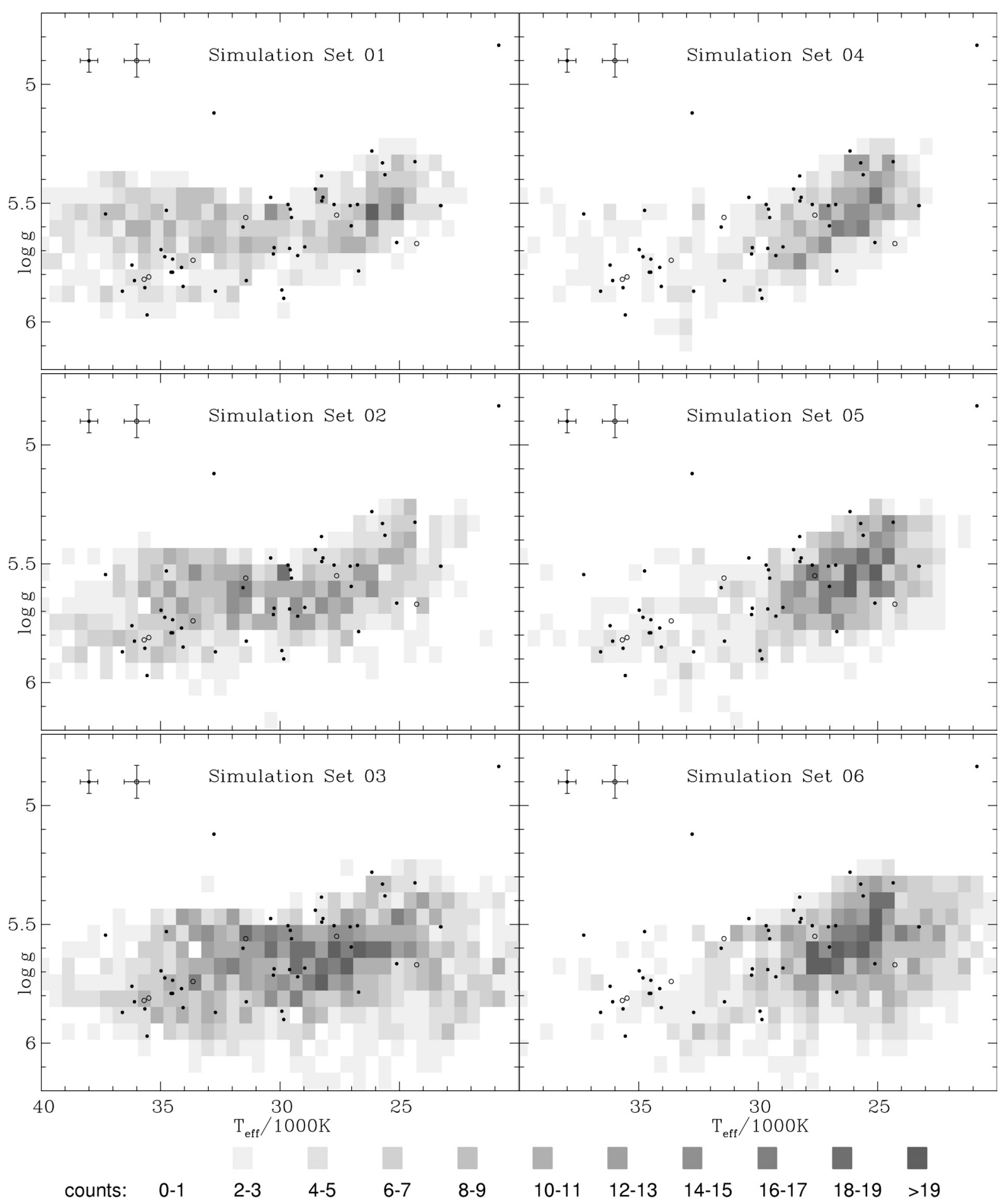

Fig. 17. Comparison of our derived values for $T_{\text {eff }}$ and $\log (g)$ (symbols and typical errors as in Fig. 6) with the simulations from HPMM. The theoretical predictions are shown as shaded $T_{\text {eff }}-\log (g)$-boxes, where a higher sdB density per box corresponds to darker shading. The grey scale is shown below the figures.

in all panels the respective leftmost track gives the worst match to the data. These tracks correspond to sets 3, 6, 9, and 12, which we have already found a trend against. Their common parameter values are $\alpha_{\mathrm{CE}}=\alpha_{\mathrm{th}}=1$, leading to the conclusion that $\mathrm{CE}$ ejection processes do not reach $100 \%$ efficiency.
In contrast to the luminosity functions derived from the Dorman et al. (1993) models, the best matching simulation sets of HPMM - as derived from our statistical analysis - are consistent with the observations at the low luminosity end of the luminosity function. It can be clearly seen, however, that they 


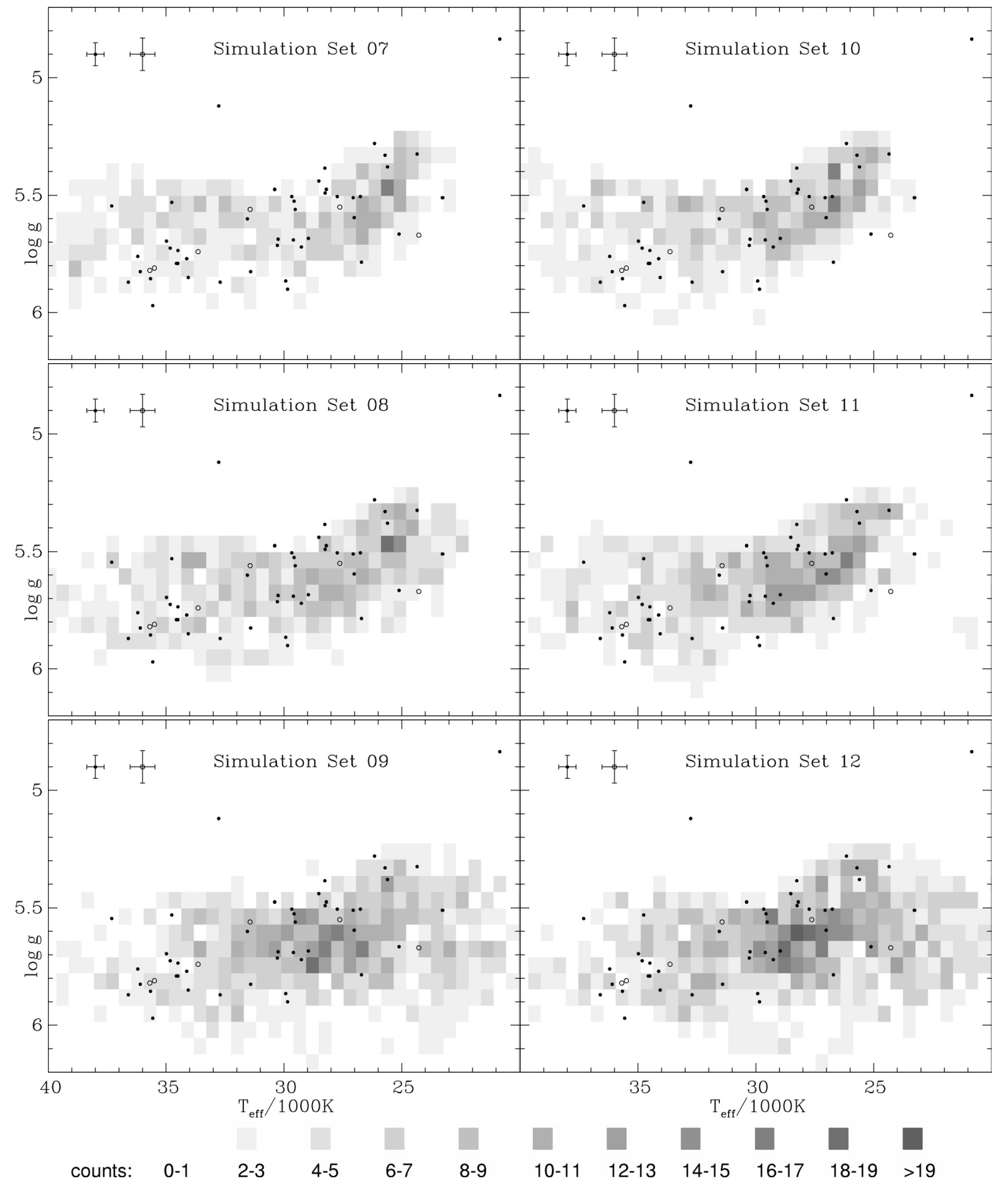

Fig. 17. Continued.

predict a smaller slope than we observe, and that even set 10 is far from reproducing the observed function. As mentioned before, any systematic errors due to LTE/NLTE or metallicity differences would not affect this statement.

\subsection{Other sdB samples}

We also investigated the sdB samples studied by Saffer et al. (1994) and Edelmann et al. (2003). A comparison to the theoretical luminosity function in the context of the single star scenario (as described for our sample in Sect. 6.1) can be made from Figs. 10 and 16. It qualitatively shows the same overall behaviour as we found for our survey, i.e. the slope of the cumulative luminosity function is the same, but the offsets of the luminosity scale are different, and the numbers of stars of too low or too high luminosity differ. We also compared these sdB samples to the HPMM simulation sets by visual inspection of the $T_{\text {eff }}-\log (g)$-plane (as described in Sect. 6.2.1) and the 

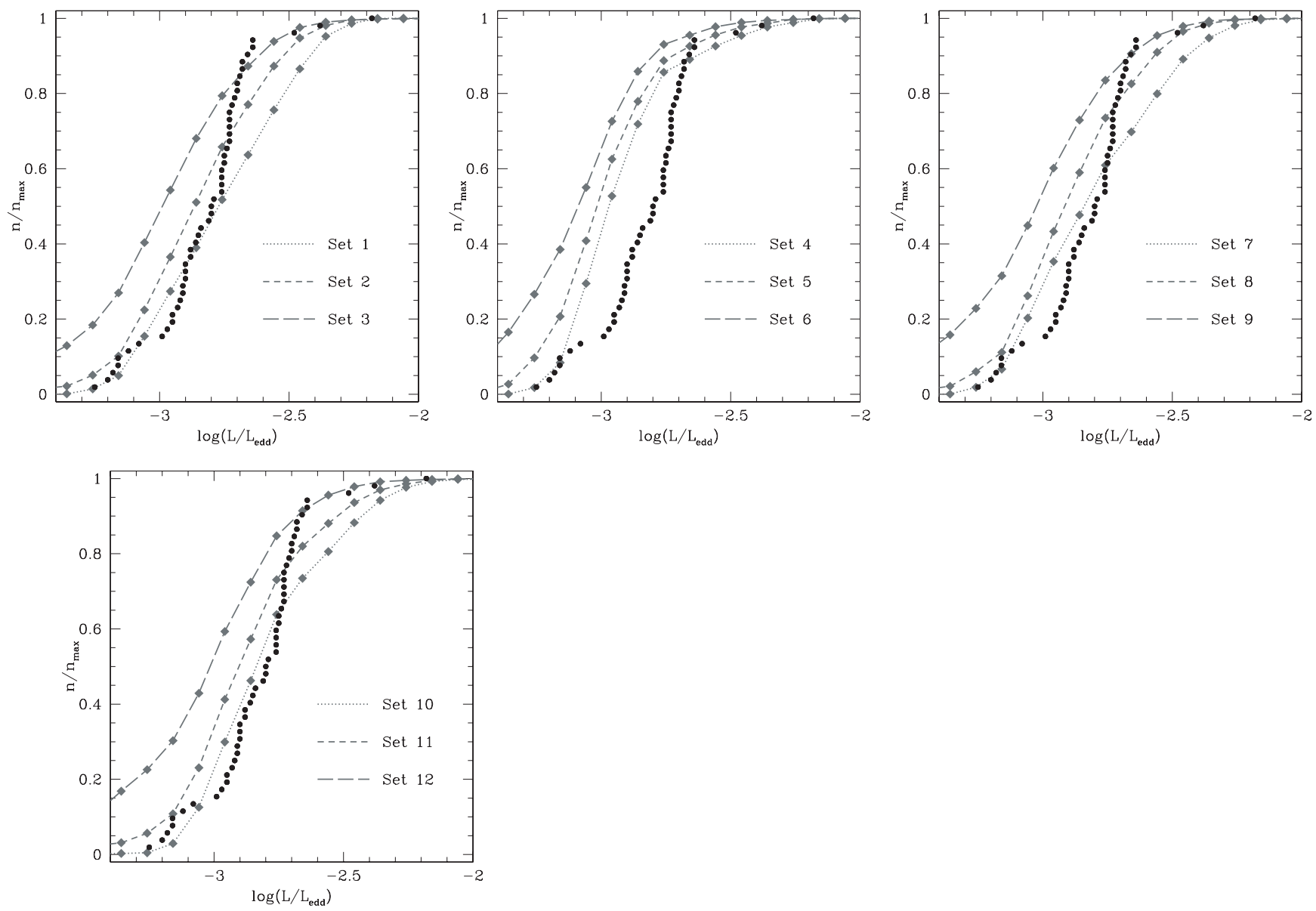

Fig. 18. Cumulative luminosity function of our sdB stars as shown in Fig. 10, along with the functions given by the HPMM simulation sets.

Table 4. Likelihood ranking of the HPMM simulation sets. The logarithmic ratio of the likelihood for the best matching set 10 to the likelihood for each set is given.

\begin{tabular}{lllllll}
\hline \hline Set & 10 & 8 & 11 & 2 & 1 & 3 \\
$\log \left(\frac{Ł_{\text {best }}}{\mathrm{t}}\right)$ & 0.0 & 1.2 & 1.5 & 2.2 & 3.5 & 3.8 \\
\hline Set & 7 & 12 & 5 & 9 & 4 & 6 \\
$\log \left(\frac{Ł_{\text {best }}}{\mathrm{Ł}}\right)$ & 4.6 & 5.0 & 6.2 & 6.2 & 7.0 & 13.9 \\
\hline
\end{tabular}

cumulative luminosity function (as described in Sect. 6.2.3). In general the result is the same as for the SPY sample. The luminosity function favors models with uncorrelated mass ratios (in particular set 4$)$, while the $T_{\text {eff }}-\log (g)$-plane rules them out. The latter favours the same models $(10,8,2,11)$ as for SPY. This directly leads us to a discussion of the current limits of $\mathrm{sdB}$ formation theories, and to suggestions for future improvements.

\section{Discussion}

To constrain the two CE ejection parameters further does not seem possible nor reasonable because of poor knowledge of $\mathrm{CE}$ ejection physics. This is also reflected in the controversial statements by HPMM and Soker \& Harpaz (2003) about the treatment of $\mathrm{CE}$ ejection processes. The latter authors criticize HPMM's criterion for CE ejection, and suggest to account for enhanced mass-loss rates by binary interaction, rather than including CE ionization energy as HPMM did.

To decide between a value of 1.2 and 1.5 for $q_{\text {crit }}$ would be of enormous interest for the predicted number of $\mathrm{sdB}$ stars that are hidden from view because they are outshone by main sequence companions of spectral types B to F. Especially the percentage of A-type main sequence stars in the Galaxy that have sdB companions is highly sensitive to $q_{\text {crit }}$, predicted to be $0.75 \%$ if $q_{\text {crit }}=1.5$, but only $0.19 \%$ if $q_{\text {crit }}=1.2$. HPMM point out that "the effects of a tidally enhanced wind can to some degree be implicitly included by using larger values of $q_{\text {crit }}$ ". This immediately suggests that various values of $q_{\text {crit }}$ may be realized in sdB formation, depending on the presence and strength of tidal wind enhancement.

Similarly, it cannot be doubted that various metallicities may play a role among the observed sdB stars, since their progenitors' ZAMS masses range from $M_{\text {ZAMS }}<1 M_{\odot}-$ corresponding to main sequence lifetimes comparable to the age of the Galaxy - to almost $M_{\mathrm{ZAMS}}=3 M_{\odot}$, having a much shorter main sequence lifetime. In addition, sdB stars exist in some halo globular clusters, and kinematic studies show that sdB stars are present in the thin disk, the thick disk, and the halo (Altmann et al. 2004). Hence, a mixture of different parameter 
configurations and population memberships may be realized in nature instead of having fixed values for all physical quantities involved.

These considerations require $\mathrm{sdB}$ formation theories to be even more complex than the HPMM simulations. In addition, the comparison of our data with the predicted cumulative luminosity functions of HPMM and Dorman et al. (1993) in the previous section can well be interpreted with the necessity of combining single star and binary formation channels to reproduce the observed sdB population. Although not compelling, one can see in Fig. 18 that the best-matching slope of the HPMM simulation sets is given by set 4 - regardless of the offset which we had immediately excluded because it does not match the observed $T_{\text {eff }}-\log (g)$-distribution. Since the slope of the cumulative luminosity function in the single star scenario (see Fig. 16) is steeper, we could remedy the discrepancy in the binary scenario (HPMM) by allowing for a contribution by a single star evolution channel, i.e. both scenarios contribute to the observed sample. An alternative possibility is that our sample is biased against stars of higher luminosity, which, if added to the function, would flatten the steep slope and probably smooth away the sharp bend at the high luminosity end. Although this seems unlikely for our sdB sample, we still have to investigate the relevance of the sdO stars in the SPY survey for the EHB evolution, since a close connection of the hydrogen-rich sdO stars to the EHB has often been claimed. Note that sdO stars have also been excluded in the investigations of other published samples.

\section{Conclusions}

We have presented the results of a spectral analysis of $76 \mathrm{sdB}$ stars from SPY. 24 objects show spectral signatures of a cool companion, which we investigated from optical and infrared photometry. The majority, if not all, of double-lined stars have main sequence companions of types $\mathrm{F}$ to $\mathrm{K}$. When focusing on helium abundances of composite sdB stars, we find no difference when compared to non-composite objects. Of the 52 single-lined stars, four show peculiar $\mathrm{H} \alpha$ profiles, possibly indicating stellar winds. The luminosity distribution of our sample is found to be in good agreement with previous studies. The tracks for luminosity evolution of single stars calculated by Dorman et al. (1993) agree in shape with the cumulative luminosity function of our stars on and above the EHB. They show, however, a slight offset in luminosity, and in addition they cannot explain the objects below the ZAEHB. The best-matching simulation sets of the binary population synthesis calculations by HPMM match the observed sdB distribution in $T_{\text {eff }}$ and $\log (g)$ very well, but they cannot reproduce the cumulative luminosity function of our stars. The mismatch is most obvious for the simulation sets using $100 \%$ efficiency of CE ejection (sets 3, 9, and 12), which also holds for a comparison with the luminosity function of other observational datasets (Saffer et al. 1994; Edelmann et al. 2003). Furthermore, there is some evidence against an uncorrelated mass distribution of the progenitor systems, which follows from the trend of rejecting sets 4,5 , and 6 .
We conclude that a combination of single star and binary formation channels would be necessary to achieve full understanding of $\mathrm{sdB}$ formation processes. In order to solve the problems that the latter still pose, future simulations are required to incorporate a more sophisticated description of the physics involved. In parallel, high-quality observational samples like SPY are necessary to enable an even better judgement of the simulated sdB population. A crucial test of theoretical calculations would be the comparison of predicted and observed fraction of radial velocity variables, i.e. stars that formed in the CE ejection channels and thus are close binaries. Since SPY was initiated to search for RV-variations, the data are well suited for such an investigation. The subsequent step will then be to determine orbital parameters of the RV-variable sdBs from SPY by measuring their radial velocity curves. First results of these projects are reported by Napiwotzki et al. (2004), and more details will be presented in Napiwotzki et al. (in prep.).

Equally important is the completion of the analysis of hot subdwarfs from SPY, i.e. the investigation of the various sdO stars observed. This class is much less homogeneous than the sdBs, and it remains to be seen whether they (or at least some of them) are intimately connected with the EHB. Increasing the sample size of $\mathrm{sdB}$ and sdO stars should then be the ultimate step. For a detailed comparison with evolutionary models, it is mandatory to improve number statistics. A huge number of sdB and sdO stars is now being discovered by sky surveys such as the Sloan Digital Sky Survey, and needs to be studied.

Acknowledgements. We express our gratitude to the ESO staff for providing invaluable help and conducting the service observations which have made this work possible. We are grateful to Zorica Salomon for providing us with various flux-calibrated sdB spectra and their atmospheric parameters. We thank Martin Altmann, Pierre Chayer, Victor Debattista, Christian Karl, Sabine Moehler, Roy Østensen, Simon O'Toole, Cristiano Porciani, Mike Reed, Alexander Stroeer, Michele Stark, Jorick Vink, and Richard Wade for fruitful discussions and important suggestions. This publication makes use of data products from the Two Micron All Sky Survey, which is a joint project of the University of Massachusetts and the Infrared Processing and Analysis Center/California Institute of Technology, funded by the National Aeronautics and Space Administration and the National Science Foundation. The exploitation of the stellar content of the Hamburg/ESO Survey has been supported by the DFG under grant Re 353/40. Work on SPY at the Dr.-Remeis-Sternwarte Bamberg is supported by the DFG under grant Na 365/2-2. T.L. acknowledges support by the Swiss National Science Foundation. R.N. acknowledges support by a PPARC advanced fellowship.

\section{References}

Allard, F., Wesemael, F., Fontaine, G., Bergeron, P., \& Lamontagne, R. 1994, AJ, 107, 1565

Aller, L. H., Appenzeller, I., Baschek, B., et al., (ed.) 1982, Landolt-Börnstein: Numerical Data and Functional Relationships in Science and Technology

Altmann, M., Edelmann, H., \& de Boer, K. S. 2004, A\&A, 414, 181

Aznar Cuadrado, R., \& Jeffery, C. S. 2002, A\&A, 385, 131

Aznar Cuadrado, R., \& Jeffery, C. S. 2001, A\&A, 368, 994 
Bessell, M. S., \& Brett, J. M. 1988, PASP, 100, 1134

Christlieb, N., Wisotzki, L., Reimers, D., et al. 2001, A\&A, 366, 898

Cox, A. N., ed. 2000, Allen's astrophysical quantities (New York: AIP Press, Springer)

D’Cruz, N. L., Dorman, B., Rood, R. T., \& O’Connell, R. W. 1996, ApJ, 466, 359

Dekker, H., D’Odorico, S., Kaufer, A., Delabre, B., \& Kotzlowski, H. 2000, in Proc. SPIE, 4008, Optical and IR Telescope Instrumentation and Detectors, ed. M. Iye; A. F. Moorwood, 534

Demers, S., Fontaine, G., Wesemael, F., Lamontagne, R., \& Irwin, M. J. 1987, in Second Conference on Faint Blue Stars IAU Coll., 95, 497

Dorman, B., Rood, R. T., \& O’Connell, R. W. 1993, ApJ, 419, 596

Dreizler, S., Heber, U., Werner, K., Moehler, S., \& de Boer, K. S. 1990, A\&A, 235, 234

Edelmann, H., Heber, U., Hagen, H.-J., et al. 2003, A\&A, 400, 939

Fontaine, G., \& Chayer, P. 1997, in The Third Conference on Faint Blue Stars, 169

Green, R. F., Schmidt, M., \& Liebert, J. 1986, ApJS, 61, 305

Hagen, H.-J., Groote, D., Engels, D., \& Reimers, D. 1995, A\&AS, 111,195

Han, Z., Podsiadlowski, P., Maxted, P. F. L., \& Marsh, T. R. 2003, MNRAS, 341, 669

Han, Z., Podsiadlowski, P., Maxted, P. F. L., Marsh, T. R., \& Ivanova, N. 2002, MNRAS, 336, 449

Heber, U. 1986, A\&A, 155, 33

Heber, U., \& Edelmann, H. 2004, in Extreme Horizontal Branch Stars and Related Objects, Kluwer, in press [arXiv: astro-ph/0405426]

Heber, U., Maxted, P. F. L., Marsh, T. R., Knigge, C., \& Drew, J. E. 2003, in Stellar Atmosphere Modeling ASP Conf. Ser., 288, 251

Heber, U., Reid, I. N., \& Werner, K. 2000, A\&A, 363, 198

Heber, U., Hunger, K., Jonas, G., \& Kudritzki, R. P. 1984, A\&A, 130,119

Iben, I. J. 1990, ApJ, 353, 215

Jeffery, C. S., \& Pollacco, D. L. 1998, MNRAS, 298, 179

Le Borgne, J.-F., Bruzual, G., Pelló, R., et al. 2003, A\&A, 402, 433

Maxted, P. F. L., Heber, U., Marsh, T. R., \& North, R. C. 2001, MNRAS, 326, 1391
McCook, G. P., \& Sion, E. M. 1999, ApJS, 121, 1

Mengel, J. G., Norris, J., \& Gross, P. G. 1976, ApJ, 204, 488

Moehler, S., Heber, U., \& Rupprecht, G. 1997, A\&A, 319, 109

Morales-Rueda, L., Maxted, P. F. L., Marsh, T. R., North, R. C., \& Heber, U. 2003, MNRAS, 338, 752

Napiwotzki, R. 2001, A\&A, 367, 973

Napiwotzki, R. 1999, A\&A, 350, 101

Napiwotzki, R. 1997, A\&A, 322, 256

Napiwotzki, R., Karl, C. A., Lisker, T., et al. 2004, in Extreme Horizontal Branch Stars and Related Objects, Kluwer, in press [arXiv: astro-ph/0401201]

Napiwotzki, R., Christlieb, N., Drechsel, H., et al. 2001, Astron. Nachr., 322, 411

Napiwotzki, R., Green, P. J., \& Saffer, R. A. 1999, ApJ, 517, 399

Ochsenbein, F., Bauer, P., \& Marcout, J. 2000, A\&AS, 143, 23

Paczyński, B. 1971, Acta Astron., 21, 1

Pauldrach, A., Puls, J., Kudritzki, R. P., Méndez, R. H., \& Heap, S. R. 1988, A\&A, 207, 123

Saffer, R. A., Bergeron, P., Koester, D., \& Liebert, J. 1994, ApJ, 432, 351

Saio, H., \& Jeffery, C. S. 2000, MNRAS, 313, 671

Salomon, Z. 2003, Diploma thesis, University of Erlangen-Nürnberg

Schlegel, D. J., Finkbeiner, D. P., \& Davis, M. 1998, ApJ, 500, 525

Soker, N., \& Harpaz, A. 2003, MNRAS, 343, 456

Stark, M. A., \& Wade, R. A. 2003, AJ, 126, 1455

Stobie, R. S., Morgan, D. H., Bhatia, R. K., Kilkenny, D., \& O'Donoghue, D. 1987, in Second Conference on Faint Blue Stars IAU Coll., 95, 493

Tutukov, A. V., \& Yungelson, L. R. 1990, SvA, 34, 57

Unglaub, K., \& Bues, I. 2001, A\&A, 374, 570

Vink, J. S. 2004, in Extreme Horizontal Branch Stars and Related Objects, Kluwer, in press [arXiv: astro-ph/0309011]

Werner, K., \& Dreizler, S. 1999, J. Computational and Applied Mathematics, 109, 65

Wisotzki, L., Koehler, T., Groote, D., \& Reimers, D. 1996, A\&AS, 115,227 